\title{
Large-scale fluctuations in the distribution of galaxies from the two-degree galaxy redshift survey
}

\author{
F. Sylos Labini ${ }^{1,2}$, N. L. Vasilyev ${ }^{3}$, and Y. V. Baryshev ${ }^{3}$ \\ 1 Centro Studi e Ricerche Enrico Fermi, via Panisperna 89 A, Compendio del Viminale, 00184 Rome, Italy \\ e-mail: Francesco.SylosLabini@roma1.infn.it \\ 2 Istituto dei Sistemi Complessi CNR, via dei Taurini 19, 00185 Rome, Italy \\ ${ }^{3}$ Institute of Astronomy, St.Petersburg State University, Staryj Peterhoff, 198504, St. Petersburg, Russia
}

Received 11 July 2008 / Accepted 5 December 2008

\begin{abstract}
We study statistical properties of galaxy structures in several samples extracted from the two-degree galaxy redshift survey (2dFRGS). In particular, we measure conditional fluctuations by means of the scale-length method and determined their probability distribution. In this way we find that galaxy distribution in these samples is characterized by large amplitude fluctuations with a large spatial extension, whose size is only limited by the sample's boundaries. These fluctuations are quite typical and persistent in the sample's volumes, and they are detected in two independent regions in the northern and southern galactic caps. We discuss the relation of the scale-length method to several statistical quantities, such as counts of galaxies as a function of redshift and apparent magnitude. We confirm previous results, which have determined by magnitude and redshift counts that there are fluctuations of about $30 \%$ between the southern and the northern galactic caps and we relate explicitly these counts to structures in redshift space. We show that the estimation of fluctuation amplitude normalized to the sample density is biased by systematic effects, which we discuss in detail. We consider the type of fluctuations predicted by standard cosmological models of structure formation in the linear regime and, to study nonlinear clustering, we analyze several samples of mock-galaxy catalogs generated from the distribution of dark matter in cosmological $N$-body simulations. In this way we conclude that the galaxy fluctuations present in these samples are too large in amplitude and too extended in space to be compatible with the predictions of the standard models of structure formation.
\end{abstract}

Key words. cosmology: observations - cosmology: large-scale structure of Universe

\section{Introduction}

In one of his seminal papers de Vaucouleurs (1970) put into a historical perspective the problem of galaxy large-scale structures and the question about the scale where galaxy distribution turns to homogeneity ${ }^{1}$. He points out that observations have first found that galaxies are not randomly distributed, then that in the fifties the same property was assigned to cluster centers, and finally that at the end of the sixties the discovery of super-clusters has still enlarged the scale of structures in the universe, thus pushing the scale where the approach to homogeneity occurs to larger and larger scales.

In the past twenty years many observations have been dedicated to the study of the large-scale distributions of galaxies (Huchra et al. 1983; Falco et al. 1999; Giovanelli \& Haynes 1993; da Costa et al. 1988; Shectman et al. 1996; York et al. 2000; Colless et al. 2001). Despite the fact that large-scale galaxy structures, of about several hundred $\mathrm{Mpc} / \mathrm{h} \operatorname{size}^{2}$, have been observed (de Lapparent Huchra \& Geller 1986; Geller \& Huchra 1989; Giovanelli \& Haynes 1993; Gott et al. 2005) to be the typical feature of the distribution of visible matter in the local universe, the statistical analysis measuring their properties has identified a characteristic scale that has only slightly

\footnotetext{
1 Note that de Vaucouleurs simply considered the scale where galaxies approaches a random distribution. A distribution can be homogeneous, i.e. with a well-defined mean density, without being random; i.e. there can be small amplitude fluctuations with weak long-range correlations.

2 We use $H_{0}=100 \mathrm{~h} \mathrm{~km} \mathrm{~s}^{-1} \mathrm{Mpc}^{-1}$ for the value of the Hubble constant.
}

changed since its discovery forty years ago in angular catalogs. This scale, $r_{0}$, was measured to be the one at which fluctuations in the galaxy density field are about twice the value of the sample density and it was indeed determined to be $r_{0} \approx 5 \mathrm{Mpc} / \mathrm{h}$ in the Shane and Wirtanen angular catalog (Totsuji \& Kihara 1969). Subsequent measurements of this scale - see e.g. Davis \& Peebles (1983), Davis et al. (1988), Park et al. (1994), Benoist et al. (1996), Norberg et al. (2001, 2002b), Zehavi et al. (2004) found a similar value, although in several samples larger values of $r_{0}$ have been found (i.e., $r_{0} \approx 6-12 \mathrm{Mpc} / \mathrm{h}$ ). This variation was then ascribed to a luminosity dependent effect - see e.g. Davis et al. (1988), Park et al. (1994), Benoist et al. (1996), Zehavi et al. (2002).

Theoretical models of galaxy formation, like the cold dark matter (CDM) one (see Peacock 1999) are able to predict the scale $r_{0}$ once it is given the amplitude and correlation properties of fluctuations of the initial conditions in the early universe. The normalization of the matter initial condition can be obtained by measuring the amplitude and correlation properties of the anisotropies of the cosmic microwave background radiation (CMBR). Then by calculating the evolution of small density fluctuations in the linear perturbation analysis of a selfgravitating fluid in an expanding universe, it is possible to predict the scale $r_{0}$ today. This turns out, in current models such as the CDM ones, to be $r_{0} \approx 5 \mathrm{Mpc} / \mathrm{h}$ (Springel et al. 2005). On scales $r<r_{0}$ models are unable to make precise predictions of the shape of the correlation function because gravitational clustering in the nonlinear regime is difficult to be treated. 
Gravitational $N$-body simulations are then used to investigate structure formation in the nonlinear phase. In addition, given that models predict, for $r>r_{0}$, a precise type of small amplitude fluctuations, it is possible to simply relate, by using the linear perturbation analysis mentioned above, the properties of fluctuations in the present matter density field to those in the initial conditions. In a certain range of scales greater than $r_{0}$, small amplitude. fluctuations should have still positive correlations. Particularly, for $r_{0}<r<r_{\mathrm{c}}$, fluctuations have very small amplitude and weak positive correlations (see Sylos Labini \& Vasilyev 2008). On even larger scales $r>r_{\mathrm{c}}$ (where this is estimated from CMBR measurements to be $r_{\mathrm{c}} \approx 100 \mathrm{Mpc} / \mathrm{h}$ ), all models predict that the matter density field presents anti-correlations that tend to zero with a (negative) power-law behavior of the type $-r^{-4}$ (Gabrielli et al. 2002; Sylos Labini \& Vasilyev 2008). This negative power-law tail corresponds in real-space to the linear dependence of the matter power-spectrum (PS) on the wave-number; i.e., $P(k) \sim k$. The former represents a behavior that can be interpreted as a consistency requirement for the properties of density fluctuations in Friedmann-Robertson-Walker models (Gabrielli et al. 2002). Because of the change in sign of the correlation function at $r_{\mathrm{c}}$, this length-scale represents the cut-off in the size of weak amplitude structures in standard models. Thus, in the regime where fluctuations are small and have weak positive correlations; i.e., for $r_{0}<r<r_{\mathrm{c}}$, the present matter-density field reflects the imprint of the initial conditions.

The fundamental test for current models of galaxy formation then concerns whether density fluctuations on large scales (i.e., $r>10 \mathrm{Mpc} / \mathrm{h}$ ) have small amplitude or not. Another important question concerns the detection of anti-correlations on scales of $r>r_{\mathrm{c}} \approx 100 \mathrm{Mpc} / \mathrm{h}$ (Sylos Labini \& Vasilyev 2008). The primary problem to be considered in this respect concerns the statistical methods used to measure the amplitude of fluctuations and the range of correlations. There has been intense debate in the past decade concerning this crucial point (Sylos Labini et al. 1998; Wu et al. 1999; Hogg et al. 2005; Gabrielli et al. 2005; Baryshev \& Teerikorpi 2005; Sylos Labini et al. 2008). Before one determines the amplitude of fluctuations in a given volume with respect to the sample density, one must have firstly tested that the former quantity is stable; i.e., that it does not depend on the sample size and/or it does not present large fluctuations in different samples containing the same type of objects. Indeed, in case the distribution presents structures and fluctuations on all scales in a given sample (i.e., it is inhomogeneous) the sample density is not a well-defined descriptor (Sylos Labini et al. 1998, 2008). In this situation all statistical quantities that are normalized to the sample density are affected by systematic effects. For this reason, prior to the characterization of fluctuations with respect to the sample density, a fundamental test consists in measuring conditional correlation properties (Gabrielli et al. 2005). It has been found that conditional statistical quantities, such as the conditional number of points in spheres, indeed show scaling properties on small scales $r<20 \mathrm{Mpc} / \mathrm{h}$, e.g., the former grows as a function of distance more slowly than the volume (Sylos Labini et al. 1998; Hogg et al. 2005; Vasilyev et al. 2006; Sylos Labini et al. 2007, 2008). This result implies that unconditional quantities are affected by systematic finite-size effects and thus do not give a reliable and meaningful estimation of correlations and amplitude of fluctuations. In this situation the length scale $r_{0}$ can be an artifact of a statistical analysis, which assumes that the sample average is a meaningful estimation of the asymptotic density; i.e., it assumes that the distribution is homogeneous and that fluctuations have a small amplitude well inside the sample volume.
While estimations of real-space correlation properties can be affected by finite-size effects, this is not the case when one counts galaxies as a function of redshift or apparent magnitude. In this case indeed one does not normalize statistical quantities to the sample average, and large fluctuations have been found both in redshift (Kerscher et al. 1999; Chiaki et al. 2003) and angular surveys (see Picard 1991; Frith et al. 2003). In particular, in a CCD survey of bright galaxies within the northern and southern strips of the $2 \mathrm{dF}$ galaxy redshift survey (2dFGRS) conclusive evidence is found of fluctuations of $\sim 30 \%$ in galaxy counts as a function of apparent magnitude (Busswell et al. 2004). Since in the angular region toward the southern galactic cap (SGC) a deficiency, with respect to the northern galactic cap (NGC) in the counts below magnitude $\sim 17$ (in the $B$ filter) was found, persisting over the full area of the APM and APMBGC catalogs, this would be evidence that there is a large void with a radius of about $150 \mathrm{Mpc} / \mathrm{h}$, implying that there is more excess largescale power than detected in the $2 \mathrm{dFGRS}$ correlation function ${ }^{3}$ (Norberg et al. 2001, 2002b) or expected in the CDM models. It is indeed evident that, because of the difference in the counts' amplitude, and thus in the sample density, between the NGC and the SGC samples, any estimation of the sample density is not stable. Thus the problems for the normalization of fluctuations amplitude to the estimation of the sample density should be studied in great detail.

In this paper we use the $2 \mathrm{dFGRS}$ to study fluctuations in galaxy distribution on large scales and to determine their statistical properties in redshift and magnitude space. Our aim is to employ statistical descriptors that do not, implicitly or explicitly, make use of the normalization of fluctuations to the sample average, thus avoiding the a-priori assumption of homogeneity inside a given sample. Thus we determine conditional statistical properties, thereby expanding our previous findings in this same survey (Vasilyev et al. 2006; Sylos Labini et al. 2009). We find that the puzzle of the coexistence of difference in densities in the NGC and SGC volumes on large spatial scales with a relatively small typical length scale of a few Mpc, can be understood as due to finite size effects in the estimation of the correlation function.

The paper is organized as follows. In Sect. 2 we discuss the 2dFGRS data and the procedure used to construct the sub samples for the statistical analysis. The methods for characterizing homogeneous and heterogeneous distributions are briefly reviewed in Sect. 3. In Sect. 4 we present the main results of the analysis and consider in detail the problems related to estimating fluctuations' amplitude normalized to the sample density. The properties of fluctuations of the matter density field predicted by theoretical models, in the linear regime, are briefly reviewed in Sect. 5. Then, to study expected nonlinear fluctuations in standard theoretical models, we analyze the properties of mock-galaxy catalogs, generated from the dark matter density fields stemming from a cosmological $N$-body simulation the Millennium Run by Springel et al. (2005). Finally in Sect. 6 we discuss the results of the analysis of the 2dFGRS samples and outline our main conclusions.

\section{The 2dFGRS samples}

The 2dFGRS (Colless et al. 2001) measured redshifts for more than 220000 galaxies in two strips in the SGC and in the NGC.

\footnotetext{
3 These statistics, as mentioned above, normalize the amplitude of fluctuations to the estimation of the sample density. From it the length scale $r_{0} \approx 6-8 \mathrm{Mpc} / \mathrm{h}$ is derived.
} 
Table 1. Main properties of the obtained VL samples. $R_{\min }, R_{\max }$ are the chosen limits for the metric distance; $M_{\min }, M_{\max }$ are the corresponding limits in the absolute magnitude; $N_{\mathrm{g}}$ is the number of galaxies in the sample.

\begin{tabular}{lccccc}
\hline \hline VL sample & $R_{\min }$ & $R_{\max }$ & $M_{\min }$ & $M_{\max }$ & $N_{\mathrm{g}}$ \\
\hline SGC400 & 100 & 400 & -20.8 & -19.0 & 29373 \\
NGC400 & 100 & 400 & -20.8 & -19.0 & 23208 \\
SGC550 & 150 & 550 & -21.2 & -19.8 & 26289 \\
NGC550 & 150 & 550 & -21.2 & -19.8 & 18030 \\
\hline
\end{tabular}

The median redshift is $z \simeq 0.1$. The apparent magnitude corrected for galactic extinction in the $b_{J}$ filter is limited to $14.0<$ $b_{J}<19.45$. The selection of the samples used in the analysis is described in detail in Vasilyev et al. (2006). Here we briefly summarize the main points.

- To avoid the effect of the irregular edges of the survey, we selected two rectangular regions: in the SGC there is a slice of size $84^{\circ} \times 9^{\circ}$ limited by $-33^{\circ}<\delta<-24^{\circ},-32^{\circ}<\alpha<$ $52^{\circ}$, while the NGC slice is smaller, i.e., $60^{\circ} \times 6^{\circ}$, with limits $-4^{\circ}<\delta<2^{\circ}, 150^{\circ}<\alpha<210^{\circ}$ (coordinates are equatorial). The solid angles are $\Omega=0.20,0.11$ steradians for the SGC and the NGC slices.

- We selected galaxies in the redshift interval $0.01 \leq z \leq 0.3$, with redshift quality parameter larger or equal to three, in order to get high quality redshifts (Hawkins et al. 2003).

- We did not use a correction for the redshift-completeness mask and for the fiber collision effects. In fact, completeness varies mostly near the survey edges, which are excluded in our sample. We assumed that fiber collisions do not make a noticeable change in the small-scale correlation properties given that we set our lower cut-off to $0.5 \mathrm{Mpc} / \mathrm{h}$, which is larger than the $0.1 \mathrm{Mpc} / \mathrm{h}$ used by Hawkins et al. (2003).

- The metric distance is usually computed as in Zehavi et al. (2002):

$R(z)=\frac{c}{H_{0}} \int_{\frac{1}{1+z}}^{1} \frac{\mathrm{d} y}{y \cdot\left(\Omega_{M} / y+\Omega_{\Lambda} \cdot y^{2}\right)^{1 / 2}}$,

where we used the standard model parameters $\Omega_{M}=0.3$ and $\Omega_{\Lambda}=0.7$, and $c$ is the light speed.

- The absolute magnitude was computed as in Zehavi et al. (2002):

$M=b_{J}-5 \cdot \log _{10}[R(z) \cdot(1+z)]-K(z)-25$,

where $K(z)$ is the $K$-correction term (Hogg et al. 2002).

- To calculate the $K$-correction $K(z)$ we used relations obtained by Madgwick et al. (2002) and we applied them as in Vasilyev et al. (2006).

- The volume-limited (VL) samples were identified by two limits in the metric distance $R_{\min }<R<R_{\max }$ and two corresponding limits in the absolute magnitude $M_{\min }$ and $M_{\max }$. In Table 1 we report the properties of the VL samples.

It is known that the completeness-mask for the survey includes the effects not only of redshift incompleteness (which varies both with field and with apparent magnitude), but also of the variations in the faint limiting magnitude across the survey regions. In order to test the robustness of our results, without correcting directly for the incompleteness using ad-hoc assumptions, we restricted the sample to the apparent magnitude limits $14.5<b_{J}<19.3$ (Hawkins et al. 2003). We report in Table 2 the characteristics of the VL with these more conservative cuts.
Table 2. As Table 1 but for the case of more conservative cuts in apparent magnitudes; i.e. $14.5<b_{J}<19.3$, were used for selecting the galaxies.

\begin{tabular}{lccccc}
\hline \hline VL sample & $R_{\min }$ & $R_{\max }$ & $M_{\min }$ & $M_{\max }$ & $N_{\mathrm{g}}$ \\
\hline SGC400 & 100 & 400 & -20.6 & -19.2 & 22872 \\
NGC400 & 100 & 400 & -20.6 & -19.2 & 18407 \\
SGC550 & 150 & 550 & -21.1 & -20.0 & 18890 \\
NGC550 & 150 & 550 & -21.1 & -20.0 & 13495 \\
\hline
\end{tabular}

As a self-consistent test, we note below that the statistical analysis in the samples constructed with less conservative cuts agree with previous determinations for what concerns the galaxy counts as a function of apparent magnitude (Norberg et al. 2002a; Busswell et al. 2004), the redshift distribution (Ratcliffe et al. 1998; Busswell et al. 2004), and the standard two-point correlation function (Norberg et al. 2001, 2002b).

\section{Statistical methods}

In this section we review the main properties of stationary stochastic point processes. These include both the ones that have a strictly positive ensemble average density and those which have it equal to zero.This discussion clarifies what the useful statistical methods are in both cases for analyzing a finite sample. A more exhaustive treatment can be found in Gabrielli et al. (2005).

\subsection{Volume average, ensemble average, and self-averaging property}

The problem of the statistical characterization of correlations and fluctuations of a stochastic distribution of points in a finite sample of volume $V$ can be rephrased as the problem of measuring volume-averaged statistical quantities. The basic issue concerns whether or not these are meaningful descriptors, i.e., whether or not they give stable statistical estimations of ensemble averaged quantities. In this respect one has to consider various problems that maybe clarified after the definition of the general probabilistic properties of stochastic point processes.

First of all, we need to define the ensemble properties. In general it is assumed that galaxy distribution is a stationary stochastic process, which means that it is statistically, translationally, and rotationally invariant; i.e., it satisfies the condition of spatial statistical isotropy and homogeneity in order to avoid special points or directions ${ }^{4}$. Stationary stochastic distributions also satisfy these conditions when they have zero average density in the infinite volume limit (Gabrielli et al. 2005).

Due to the assumption of ergodicity (i.e., the ensemble average is equal to the infinite volume average), the existence of a well-defined average density implies that, for a single realization of the mass distribution, the following limit is well-defined:

$\lim _{R \rightarrow \infty} \frac{1}{V\left(R ; \boldsymbol{x}_{0}\right)} \int_{V\left(R, \boldsymbol{x}_{0}\right)} n(\boldsymbol{r}) \mathrm{d} \boldsymbol{r}=n_{0}, \forall \boldsymbol{x}_{0}$.

where $V\left(R, x_{0}\right) \equiv 4 \pi R^{3} / 3$ is the volume of the sphere of radius $R$, centered on an arbitrary point $\boldsymbol{x}_{0}$. The constant $n_{0}$ is strictly positive for homogeneous (or uniform ${ }^{5}$ ) distributions and zero for

\footnotetext{
4 Because of the hypothesis of statistical stationarity, statistical quantities generally depend only of the scalar distance between points.

5 To avoid confusion a stationary stochastic point process with a positive ensemble average density is sometimes denoted as uniform and
} 
inhomogeneous ones (e.g. fractals). It is then clear that all statistical quantities that are normalized to $n_{0}$ are meaningless when this is equal to zero in the ensemble average or in the infinite volume limit.

Keeping these mathematical properties in mind we have to consider the situation occurring when in a finite sample, of size $\sim V^{1 / 3}$, the distribution is not homogeneous. In this case the estimator of the average mass density gives a large relative error with respect to the ensemble value making it systematically biased. This implies that only statistical averages conditioned to the fact that the origin of coordinates is a point of the set are well-defined.

Clearly a distribution can be inhomogeneous up to a length scale $\lambda_{0}{ }^{6}$ and homogeneous for $r>\lambda_{0}$. Then for $r<\lambda_{0}$ the distribution is characterized by large fluctuations, and the average density is not a well-defined quantity if it is estimated in samples of size $V^{1 / 3}<\lambda_{0}$. Instead density fluctuations are small for $r>\lambda_{0}$ and the sample density converges to the asymptotic (or ensemble) value when the sample volume is such that $V^{1 / 3}>\lambda_{0}$. The precise behavior of this convergence is determined by the (weak) two-point correlation properties of the distributions, and the convergence will be slower when correlations are long-range (Gabrielli et al. 2005).

\subsection{Probability distribution of conditional fluctuations}

In Sylos Labini et al. (2008) we have introduced the scale-length (SL) analysis. This consists in determining the number $N\left(r ; R_{i}\right)$ of galaxies in spheres of radius $r$, centered on the $i$ th galaxy ${ }^{7}$ whose distance from the origin is $R_{i}$; that is,

$N\left(r ; R_{i}\right)=\int_{V\left(r ; R_{i}\right)} n(s) \mathrm{d} s$,

where the integral is performed over the spherical volume $V\left(r ; R_{i}\right)$ of radius $r$ centered on the $i$ th galaxy at distance $R_{i}$ from the origin, and $n(s)$ is the microscopic number density, which is a function of the space position $\boldsymbol{s}$.

When Eq. (4) is averaged over the whole sample, it gives an estimate of the average conditional number of galaxies in spheres of radius $r$

$\overline{N(r)}=\frac{1}{M(r)} \sum_{i=1}^{M(r)} N\left(r ; R_{i}\right)$,

where the sum is extended to the $M(r)$ galaxies, the $i$ th at radial distance $R_{i}$ from us, whose separation from the boundaries of the sample is less than or equal to $r$. In this way when $r$ grows the number of values $M(r)$ over which the mean in Eq. (5) is performed decreases with $r$, because only those galaxies for which the sphere is fully included in the sample volume are considered as centers.

This estimator, known as the full-shell estimator (Sylos Labini et al. 1998; Kerscher 1999; Gabrielli et al. 2005), has the advantage of making the weakest a-priori assumptions about the properties of the distribution outside the sample volume. Indeed one may use incomplete spheres by counting the number of galaxies inside a portion of a sphere and weighting this for the corresponding volume of the spherical portion

nonuniform when this is equal to zero. Both are statistically homogeneous stochastic processes (Gabrielli et al. 2005).

6 See below for a precise definition.

7 This is thus a conditional quantity.
(Kerscher 1999). However, this method implicitly uses the assumption that what is inside the incomplete sphere is a statistically meaningful estimate of the distribution in the whole spherical volume. This is incorrect when the distribution presents large fluctuations. For example in the part of a spherical volume that lies outside the sample boundaries, there can be a void or a largescale structure and in this situation the weighted estimation is biased (Gabrielli et al. 2005). When the full-shell estimator is used, one should consider that there is an intrinsic selection effect related to the geometry of the samples, which are small portions of spheres. When $r$ is large only the more distant part of the sample is explored by the volume average (Gabrielli et al. 2005; Sylos Labini et al. 2008). Indeed, for large-sphere radii, $M(r)$ decreases and the location of the galaxies contributing to the average in Eq. (5) is mostly placed at radial distance in the range $\sim\left[R_{\min }+r, R_{\max }-r\right]$ from the radial boundaries of the sample at $\left[R_{\min }, R_{\max }\right]$. Given the geometry of the samples for large $r$, galaxies contributing to $M(r)$ will also lie toward the center of the spherical portion.

When Eq. (5) scales as

$\overline{N(r)} \sim r^{D}$

and $D=3$, the distribution is homogeneous, while for $D<3$ it has long-range power-law correlations (Gabrielli et al. 2005). The scaling of Eq. (6) with $D<3$ can be interpreted as the signature that the distribution is a fractal; however there are point distributions that, by construction, are not fractal objects but which may exhibit a scaling of the type given by Eq. (6) (see Gabrielli et al. 2005).

From Eq. (6) we obtain that, in general, for $D<3$ the conditional density scales as

$\overline{n(r)}=\frac{\overline{N(r)}}{V(r)} \sim r^{D-3}$

in this situation the average density is not a well defined quantity and the sample density is depends on the sample size; i.e., it does not give a meaningful estimation of the ensemble average density.

When a distribution is fractal (or generally inhomogeneous) on small scales and homogeneous on large scales, then we can identify the homogeneity scale $\lambda_{0}$ to be the scale such that (Gabrielli et al. 2005)

$\overline{N(r)} \sim r^{3}, \forall r>\lambda_{0}$.

Depending on the details of the crossover from the strongly correlated regime at $r<\lambda_{0}$ to the weakly correlated one at $r>\lambda_{0}$ different definitions of $\lambda_{0}$ can be adopted, but they all satisfy the condition given by Eq. (7). For instance one may define such a scale to be the one at which the rms fluctuation have twice the value of the average density. If strong clustering occurs only for $r<\lambda_{0}<V^{1 / 3}$ it is required to make the statistical analysis suitable to describe large fluctuations in this range of scales. Instead for $\lambda_{0}<r<V^{1 / 3}$ usual unconditional quantities are well-defined and their convergence to the ensemble values can be studied.

The estimator defined by Eq. (5) gives the first moment (i.e., the average number of points in spheres of radius $r$ ) of the PDF of conditional fluctuations $f(N ; r)$ computed, at fixed $r$, from the values $\left\{N\left(r ; R_{i}\right)\right\}_{i=1, \ldots, M(r)}$. This is generally different from the PDF of unconditional fluctuations - considered by, e.g., Saslaw (2000) - both for homogeneous and inhomogeneous distributions, the difference being more important in the former case. 
When a distribution becomes homogeneous; i.e., Eq. (7) is satisfied, the PDF is expected to converge in a finite volume to a Gaussian function ${ }^{8}$ (Gabrielli et al. 2005); i.e.,

$f\left(N ; r \gg \lambda_{0}\right) \simeq \frac{1}{\sqrt{2 \pi \overline{\Sigma^{2}(r)}}} \exp \left(-\frac{[N(r)-\overline{N(r)}]^{2}}{2 \overline{\Sigma^{2}(r)}}\right)$,

where $\overline{\Sigma^{2}(r)}$ is the estimation of the variance of the random variable $N(r)$ (see below).

The second moment of $f(N, V)$ gives the conditional variance. For inhomogeneous distributions, this is such that

$\overline{\delta(r)^{2}} \equiv \frac{\overline{\Sigma^{2}}(r)}{\overline{N(r)}^{2}}=\frac{\overline{N(r)^{2}}-\overline{N(r)}^{2}}{\overline{N(r)}^{2}} \sim 1$,

where the last equality means that fluctuations are persistent (Gabrielli \& Sylos Labini 2001). On the other hand, for homogeneous distributions with any kind of small-amplitude correlations, we find that (Gabrielli \& Sylos Labini 2001)

$\overline{\delta(r)^{2}} \ll 1$.

When the sample density $n_{\mathrm{S}}$ is well-defined; i.e., it does not depend on the sample volume, one may define the reduced twopoint correlation function that can be written as (Gabrielli et al. 2005)

$\xi(r)=\frac{1}{4 \pi r^{2}} \frac{\mathrm{d} \overline{N(r)}}{\mathrm{d} r} \frac{1}{n_{\mathrm{S}}}-1$.

In this case the homogeneity scale can be, for instance, defined as the scale beyond which $\xi(r)<1$, or the scale such that $\delta^{2}\left(\lambda_{0}\right)=1$ (see Gabrielli et al. 2005).

\section{Results from the 2dFGRS}

In this section we present the results of the statistical analysis of the VL samples of the 2dFGRS catalog discussed in Sect. 2. We start by presenting the SL analysis to then move to the description of the determination of the PDF of conditional fluctuations. Furthermore, we consider its first moment; i.e., the average number of points in spheres. To illustrate the usefulness of the SL analysis, we consider its relation to the counts of galaxies as a function of radial distance and of apparent magnitude. This allows us to discuss in detail the relation between small scale twopoint correlations and large scale properties of fluctuations in the galaxy density field. We then consider the finite-size effects that systematically affect the determination of fluctuations amplitude normalized to the sample density. In addition we compute the average of the SL determinations $\left\{N\left(r ; R_{i}\right)\right\}_{i=1 . . M(r)}$ in bins of radial distance. The comparison of the behaviors in the NGC and SGC slices allows us to place a lower limit on the homogeneity scale.

\subsection{The scale-length analysis}

In Figs. 1-4 the behavior of the SL analysis is shown in the four $2 \mathrm{dFGRS}$ samples we considered. One may note that in all cases there are large density fluctuations in the correspondence of the location of galaxy large-scale structures. In Fig. 5 a threedimensional plot is shown of the same SL analysis reported in

\footnotetext{
8 This is clearly the case if the number of points is large enough, otherwise the PDF is described by the Poisson distribution.
}
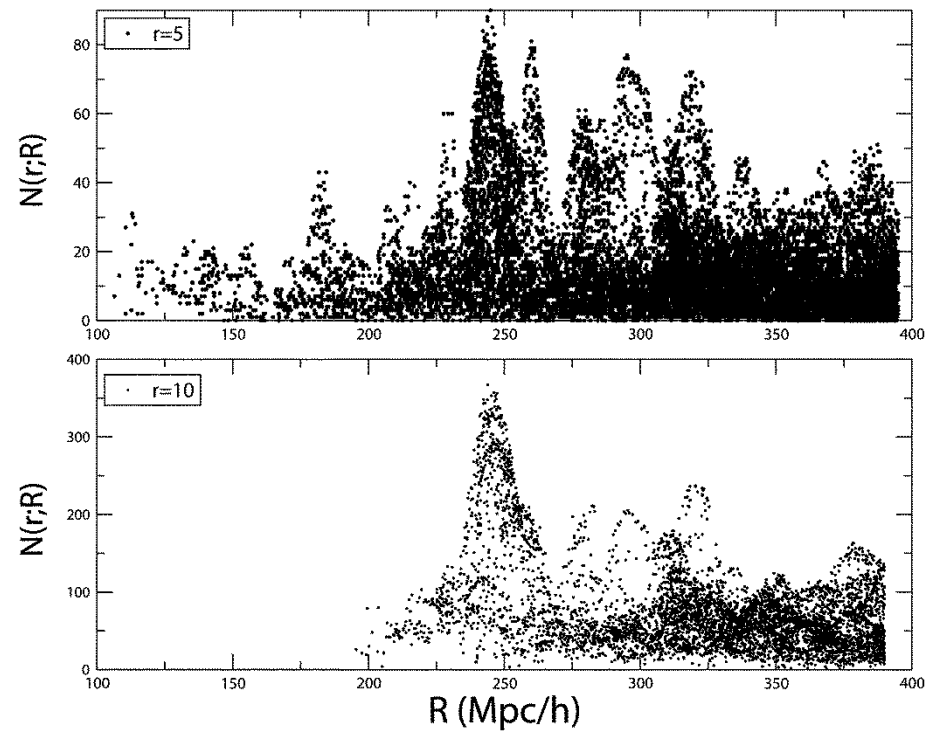

Fig. 1. From top to bottom the SL analysis for the sample NGC400 with $r=5,10 \mathrm{Mpc} / \mathrm{h}$.
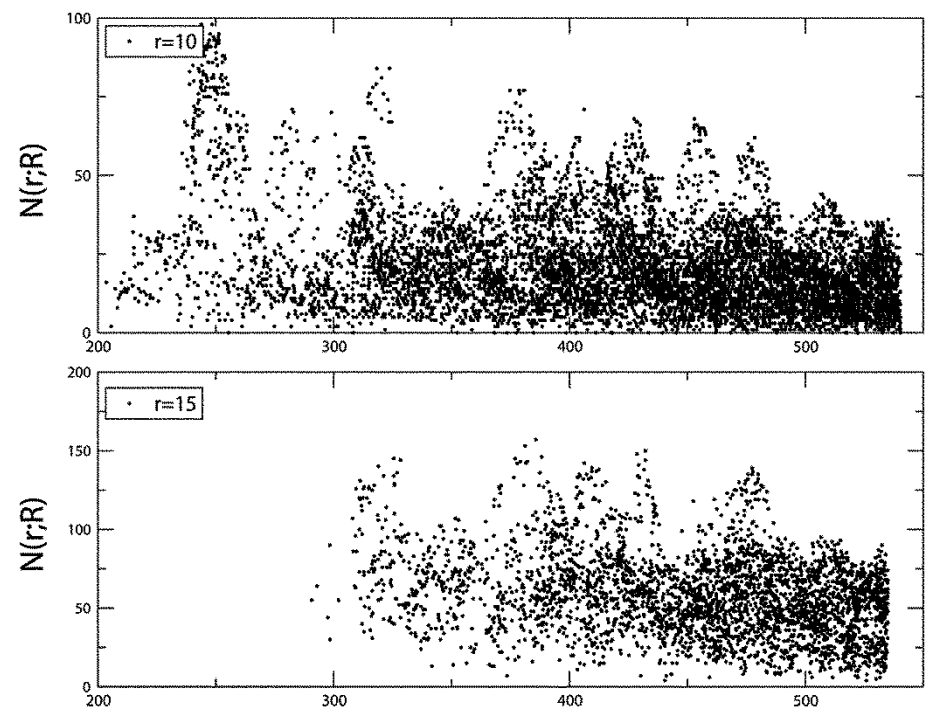

Fig. 2. The same as Fig. 1 but now for the sample NGC550 with $r=$ $10,15 \mathrm{Mpc} / \mathrm{h}$.

Fig. 4. One may see how well structures are identified by this analysis.

The number of points $M(r)$ over which $N\left(r ; R_{i}\right)$ is computed, as a function of the sphere radius $r$ is shown in Fig. 6: when the sphere radius $r$ gets larger, the $M(r)$ decreases quite rapidly. This is due to the geometrical selection effect previously discussed. In addition, the solid angle of the SGC slice is twice that of the NGC slice, and thus for $r>20 \mathrm{Mpc} / \mathrm{h}$, there are more center points in the SGC samples than in the NGC ones.

Let us briefly discuss the main features that we detect in the various samples 9

- NGC400: there are large structures, which transversely cross the sample, at about $240 \mathrm{Mpc} / \mathrm{h}$ and at 260, 270, 290 and $320 \mathrm{Mpc} / \mathrm{h}$. All have approximately the same thickness of about $30-40 \mathrm{Mpc} / \mathrm{h}$. When the sphere radius is increased to $r=10 \mathrm{Mpc} / \mathrm{h}$ the most prominent structure remains the one

\footnotetext{
9 Note that, for instance, Eke et al. (2004); Einasto et al. (2006) used different methods to identify the same structures we observe.
} 

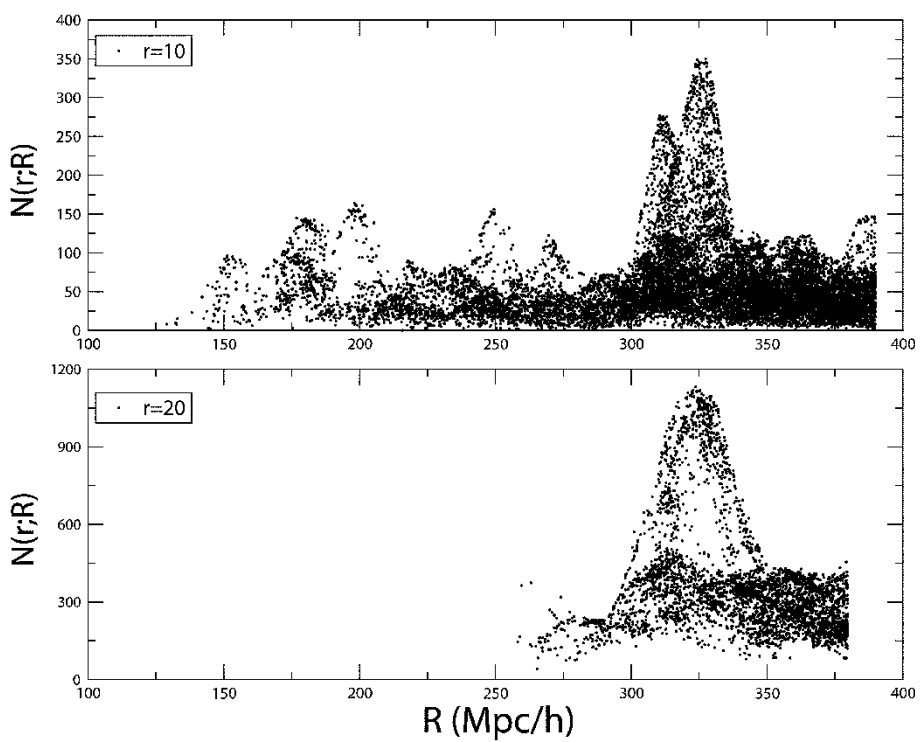

Fig. 3. The same as Fig. 1 but now for the sample SGC400 with $r=$ $5,10,20 \mathrm{Mpc} / \mathrm{h}$.
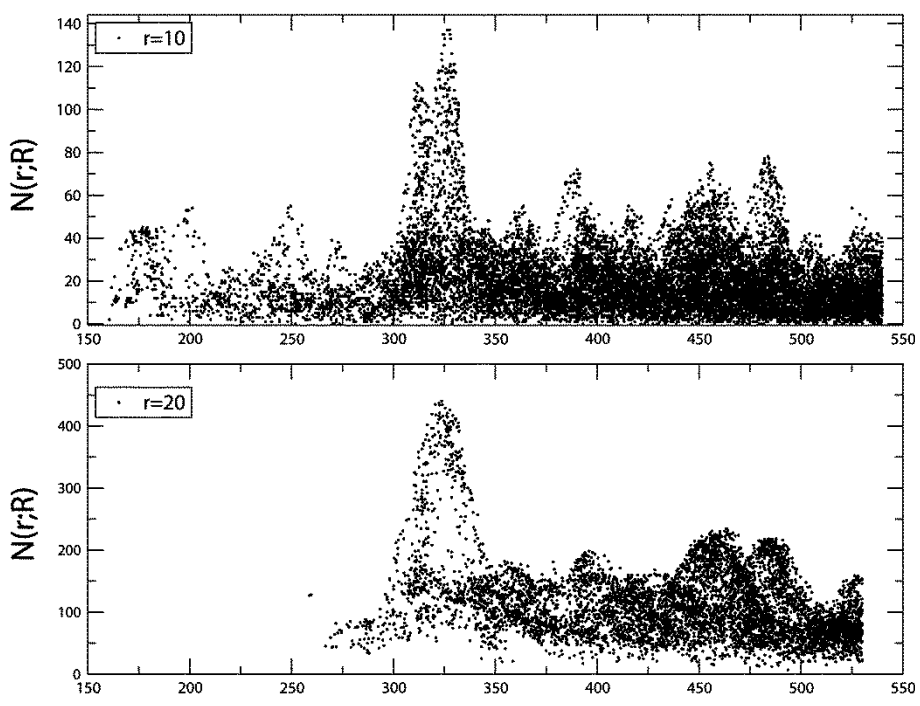

Fig. 4. The same as Fig. 1 but now for the sample SGC550 with $r=$ 10, 20, $30 \mathrm{Mpc} / \mathrm{h}$.

at about $250 \mathrm{Mpc} / \mathrm{h}$, which is not sampled anymore when $r=15 \mathrm{Mpc} / \mathrm{h}$. This is due to the sphere centers being located toward the faraway boundaries of the sample, because of the geometrical selection effect discussed previously. For $r=$ $15 \mathrm{Mpc} / \mathrm{h}$, although only a few points (i.e $M \approx 3000$ ) are effectively considered as sphere centers, density fluctuations are still large, determining variation of a factor four in the determination of the number of points in spheres at different radial distances.

- NGC550: the structure at $R \approx 250 \mathrm{Mpc} / \mathrm{h}$ is clearly visible even in this sample, and other structures are present at larger radial distances $R>350 \mathrm{Mpc} / \mathrm{h}$. Fluctuations are still large up to the largest sphere radius $r=25 \mathrm{Mpc} / \mathrm{h}$ where however the number of centers rapidly decreases for the geometrical selection effect discussed in Sect. 3.

- SGC400: for sphere radius $r=10 \mathrm{Mpc} / \mathrm{h}$ the situation is similar to the NGC400 case, except for the fact that the radial distances corresponding to the large variations in the density field are different. This shows that large-scale structures and the corresponding large fluctuations detected by the SL analysis are quite typical of the galaxy distribution. There are two prominent large-scale structures at radial distance of the order of $R \sim 320 \mathrm{Mpc} / \mathrm{h}$. Finally for $r=20 \mathrm{Mpc} / \mathrm{h}$ the sample is dominated by one of the two structures just mentioned, which corresponds to a variation of order five in $N(r ; R)$.

- SGC550: a structure at $R \approx 320 \mathrm{Mpc} / \mathrm{h}$ with thickness of about $40 \mathrm{Mpc} / \mathrm{h}$ is present inside this sample as well. In addition other structures are visible for $R>400 \mathrm{Mpc} / \mathrm{h}$. For the largest sphere radius $r=30 \mathrm{Mpc} / \mathrm{h}$, where only a few points are considered as sphere centers, fluctuations in $N(r ; R)$ are of order five and they are due to structures located at radial distances $R>380 \mathrm{Mpc} / \mathrm{h}$.

In summary galaxy distribution in these samples is characterized by several large scale structures which cross their volumes. These structures are typical and persistent; i.e., they are detected at different radial distances and in the two different directions toward the SGC and NGC. They correspond in the SL analysis to large fluctuations in the density field: the thickness of the structures is partially an intrinsic property and partially due to the "Finger of God" effect - the redshift space extension of large clusters and super-clusters - convolved with the sphere size used to measure the conditional density. Typical velocity dispersions are up to $\sim 10^{3} \mathrm{~km} \mathrm{~s}^{-1}$, although Eke et al. (2004) conclude that the typical velocity dispersion for $2 \mathrm{dF}$ groups and clusters is $260 \mathrm{~km} \mathrm{~s}^{-1}$, which only corresponds to $2.6 \mathrm{Mpc} / \mathrm{h}$ in an additional radial extension. Thus while peculiar velocities can increase the size of radial structures, their effect cannot explain the whole range of distances which characterize the observed structures.

The maximum allowed sphere radius we considered, which, as discussed, is set by the geometry of the samples, is $r=$ $30 \mathrm{Mpc} / \mathrm{h}$. For this value of $r$ we find fluctuations of order four in $N(r ; R)$ and this allows us to conclude that the homogeneity scale is certainly larger than $40 \mathrm{Mpc} / \mathrm{h}$. In what follows we reinforce this conclusion by considering suitable statistical measurements.

\subsection{Probability distributions of conditional fluctuations}

We now turn to the discussion of the PDF of conditional fluctuations The behaviors in the various samples are shown in Figs. 7-10. We limit the discussion to the case where the number of determinations $\left\{N\left(r, R_{i}\right)\right\}_{i=1, . ., M(r)}$ at fixed sphere radius $r$ is larger than few thousands points. For smaller numbers the measurement is affected by weak statistics and by finite-size effects, thus not leading to a statistically robust result. As discussed previously, when the sphere radius increases, there is a decrease in the number of centers, and thus a for large $r$, whose precise value is determined by the geometry of each specific sample, the measurement is affected by large statistical and systematic effects (see Fig. 6).

The first point to note is that in all cases the maximum of $f(N, r)$ is statistically stable; i.e., it does not change when it is computed in the whole sample or in two non-overlapping sub samples with equal volume (each half of the sample volume) at small (sub-sample $S_{1}$ ) and at large (sub-sample $S_{2}$ ) radial distance (see Figs. 7 and 9).

The tail for large values of $N$ is instead affected by the different fluctuations which are present in different sub-volumes. The trend is obvious: the larger the fluctuations of $N(r ; R)$ the more extended toward high $N$ values is the tail of $f(N, r)$. In the deepest samples, e.g. SGC550, there is a single structure that dominates the distribution. However this is placed in the middle of the 


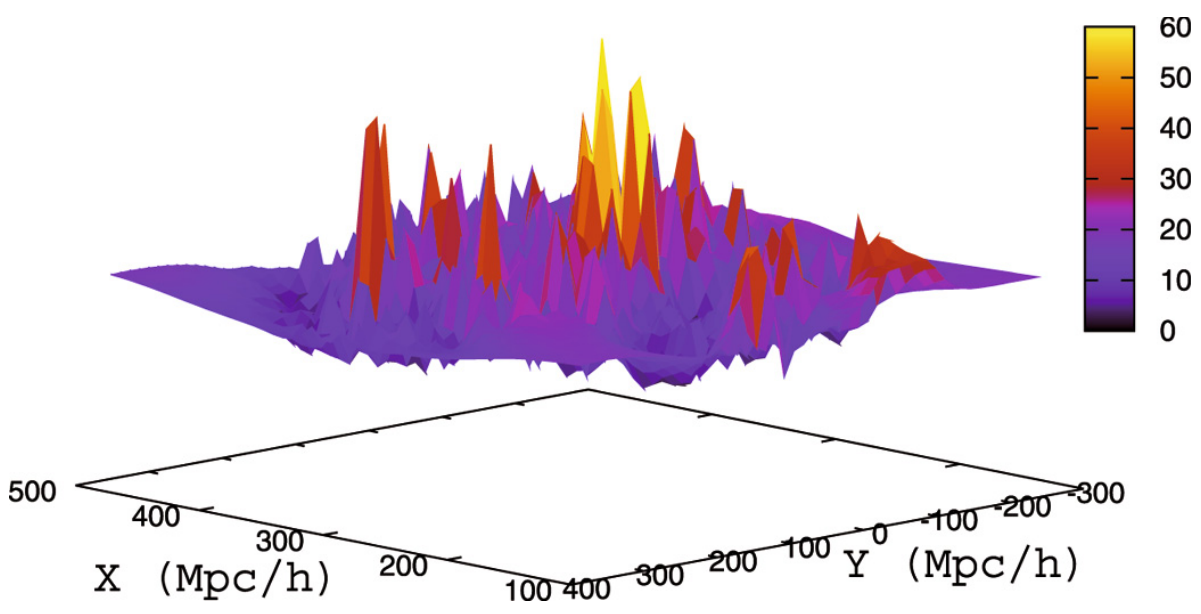

60 ple. On the $X$ and $Y$ axes the coordinate of the center of a sphere of radius $r=10 \mathrm{Mpc} / \mathrm{h}$ (centered on a galaxy) is reported and on the $Z$ axis the number of galaxies inside it. The mean thickness of this slice is about $50 \mathrm{Mpc} / \mathrm{h}$. Large fluctuations in the density field traced by the SL analysis are located in the correspondence of large-scale structures.

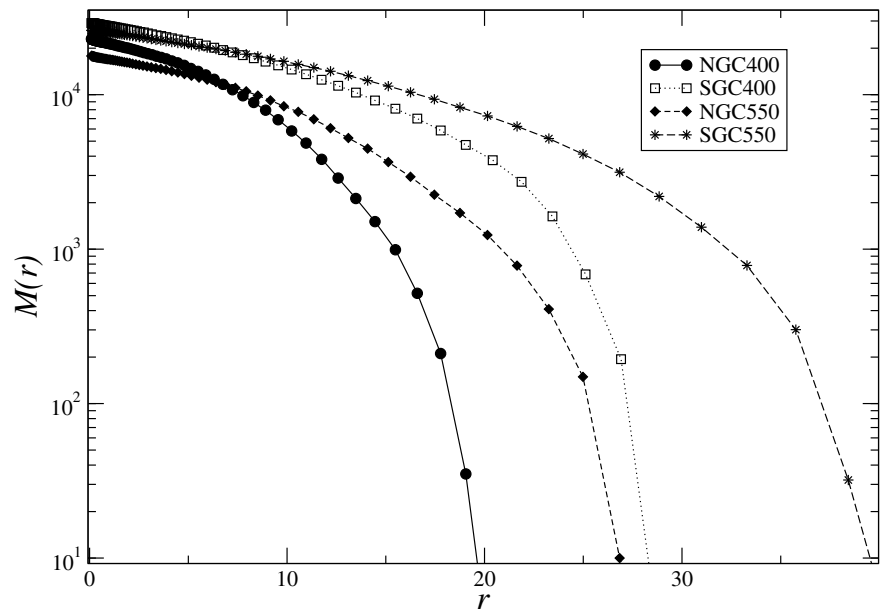

Fig. 6. Number of center-points $M(r)$ as a function of the sphere radius $r$ in the various samples considered.

sample and for this reason, apparently, there is no a systematic difference in the PDF when this is computed in the two half volumes of the sample, one nearby and one far-away. However, it is clear that in this situation the shape of the PDF will be strongly affected by this fluctuation. Indeed, in each sub sample, for the largest sphere radius $r$ we find that $f(N, r)$ is systematically distorted with respect to smaller sphere radii. This is because the volume average cannot explore the full sample properly because of the geometrical selection effect which, as discussed, is present in the determination of $N(r ; R)$. To properly determine the PDF on scales $r>20 \mathrm{Mpc} / \mathrm{h}$ larger samples are thus required.

In all cases the PDF is systematically different from a Gaussian function, except for the case of NGC550 for which there are the weakest statistics, and it is characterized by a long $N$ tail which is directly related to the large scale structures present in these samples. In addition the PDF differs in different samples, especially for $r>10 \mathrm{Mpc} / \mathrm{h}$. This implies that, because of the weak statistics and small volumes, a clean determination of the PDF is impossible. For this reason we limit our discussion in what follows to the first moment of the PDF, leaving the determination of the second moment to the other samples. for instance those of the SDSS, where spatial volumes will be larger (Sylos Labini et al. 2008).
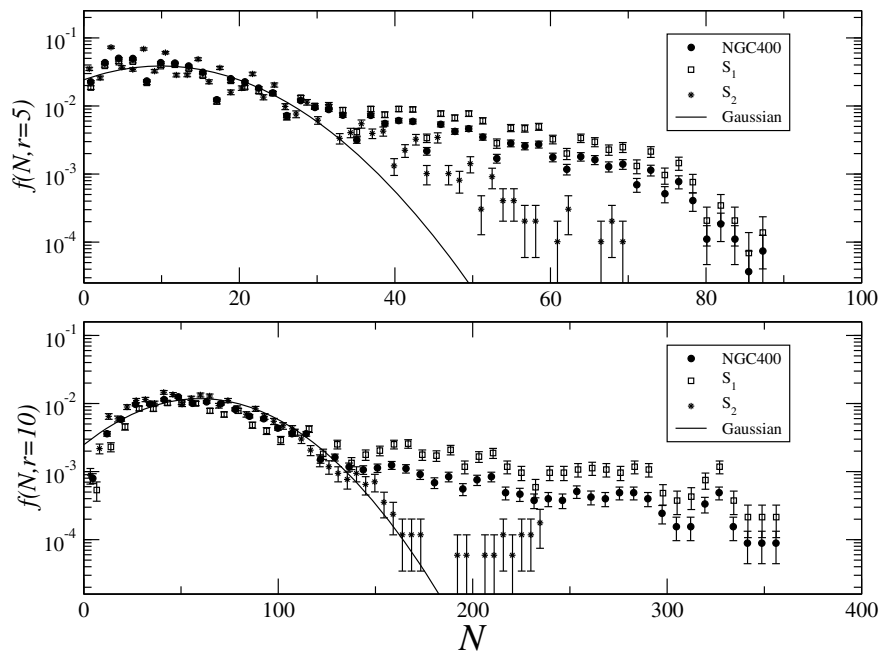

Fig. 7. Probability density function $f(N, r)$ of the values $\left\{N\left(r ; R_{i}\right)\right\}_{i=1, \ldots, M(r)}$ for NGC400 in the whole sample and the best fit with a Gaussian function. The different sphere radius $r$ is reported on the $y$-axis. The behavior in two non-overlapping sub samples (labeled as $S_{1}$ and $S_{2}$ is also reported - see text for details. Poisson error bars are shown as a reference.

In Fig. 11 we show the collapse plot of the PDF in the various samples and for the different sphere radius considered. A rough fit of the large $N$ tail is given by

$f(N, r) \sim N^{-3}$.

However, this result requires better samples before being confirmed.

\subsection{Conditional average number of galaxies in spheres}

In Fig. 12 we show the determination of the whole-sample, average conditional number of points in spheres; i.e., Eq. (5). Given that the PDF in all samples are statistically stable; i.e., they do not show important systematic differences in different subvolumes, the full sample volume average provides a meaningful statistical quantity. These behaviors are the same as the ones found by Vasilyev et al. (2006) for the average conditional density. We find that in all samples,

$\overline{N(r)}=B r^{D}$, 

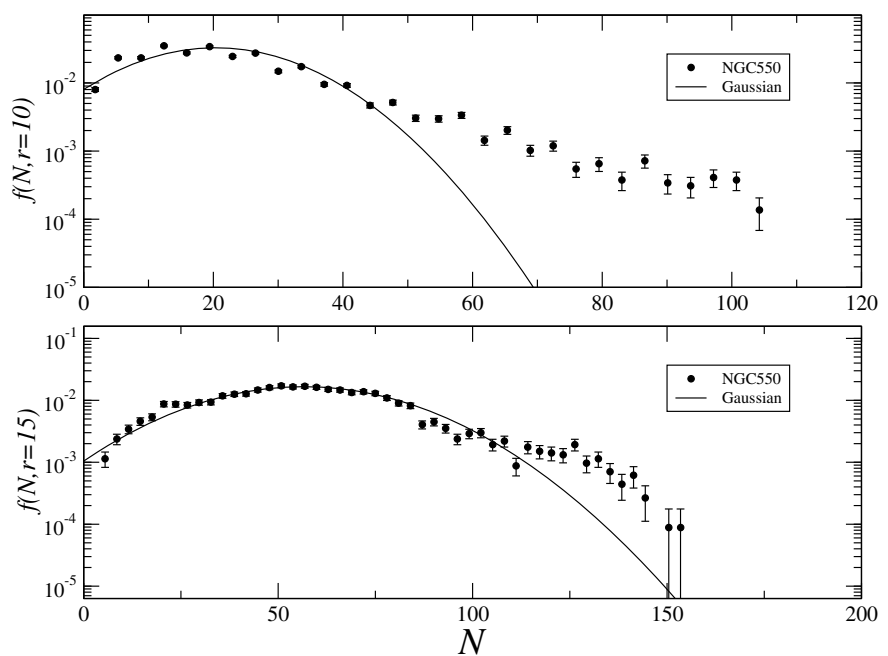

Fig. 8. The same as in Fig. 7 for the sample NGC550 with $r=$ $10,15 \mathrm{Mpc} / \mathrm{h}$. For $r=15 \mathrm{Mpc} / \mathrm{h}$ there are only 3765 determinations.

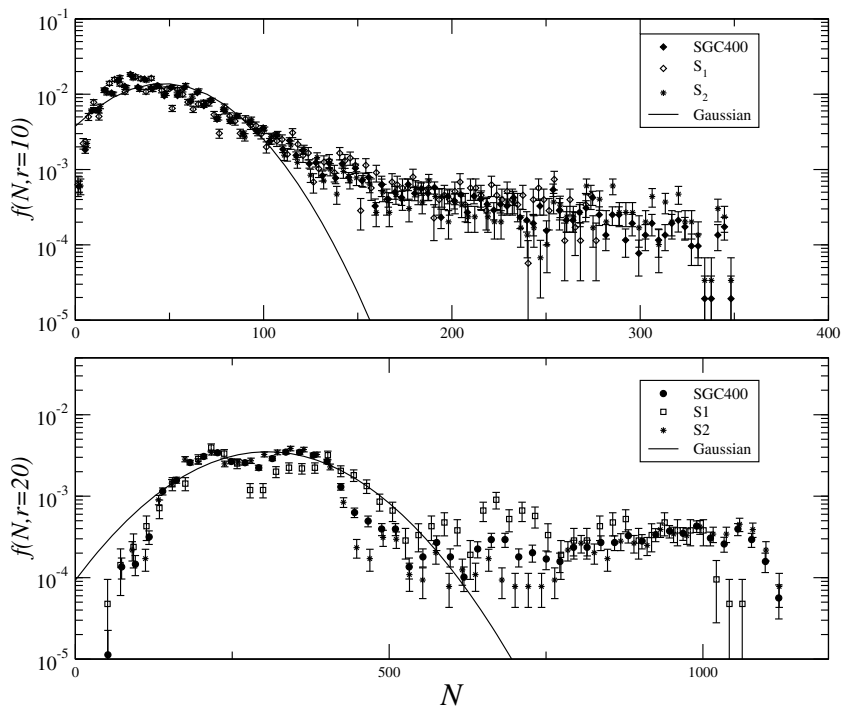

Fig. 9. The same as in Fig. 7 for the sample SGC400 with $r=$ 5, 10, $20 \mathrm{Mpc} / \mathrm{h}$.

with $D=2.2 \pm 0.1$ and $B$ a constant. This result is statistically robust up to $r=30 \mathrm{Mpc} / \mathrm{h}$ although for the SGC550 sample the analysis can reach, with a weaker statistics, $r \sim 40 \mathrm{Mpc} / \mathrm{h}$. In all cases, however, the determinations for scales $r>20 \mathrm{Mpc} / \mathrm{h}$ are done on a relatively small number of points (see Fig. 6), so they can be subject to systematic fluctuations. The amplitude between the NGC400 and SGC400 samples (which have higher number density than that of the deeper samples) differs for about $\sim 30 \%$ (see Fig. 13). This is due to the different fluctuations, which are present in the different volumes, and this implies that the samples are not large enough to precisely determine the amplitude. This difference is reflected in the number counts as a function of apparent magnitude as we discuss below. In the NGC550 and SGC550 samples, although the samples volume is larger, the constant $B$ in Eq. (12) varies $20 \%$ (see Fig. 13).

To briefly discuss the determination of the constant prefactor $B$ in Eq. (12) (Joyce \& Sylos Labini 2001; Gabrielli et al. 2005) and the normalization of the conditional average density in different VL samples one should consider the joint conditional probability of finding a galaxy of luminosity $L$ at distance $r$ from another galaxy; i.e., the (ensemble) conditional average
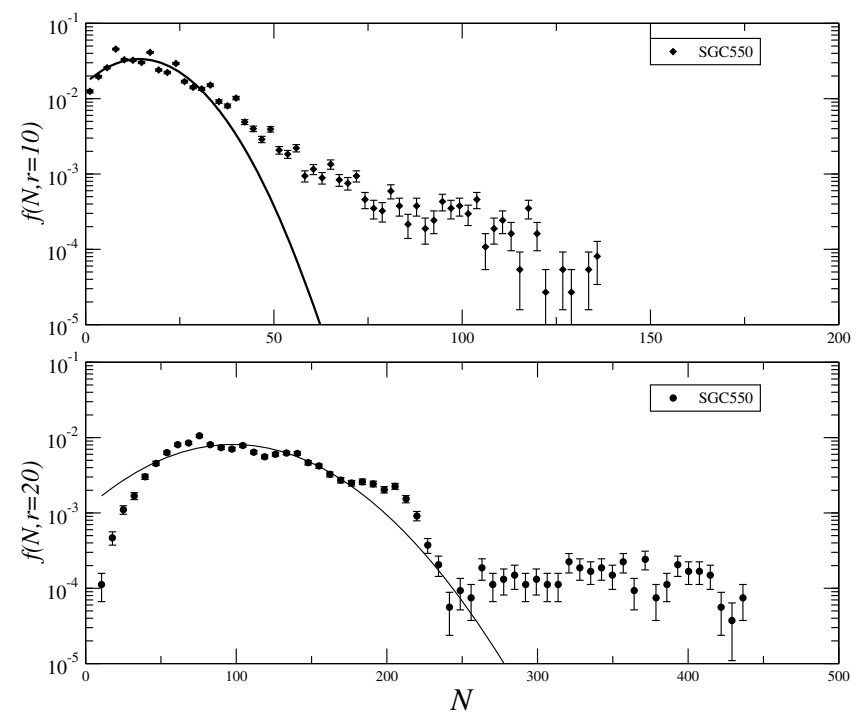

Fig. 10. The same as in Fig. 7 for the sample SGC550 with $r=10$, $20 \mathrm{Mpc} / \mathrm{h}$.

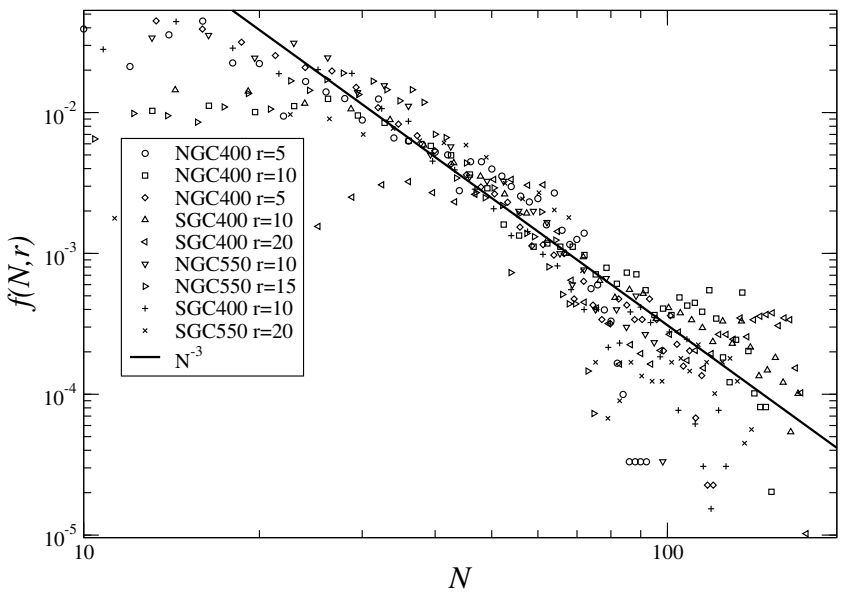

Fig. 11. Collapse plot of the $f(N, r)$ in the various samples and for the different sphere radius considered. The normalization of the different samples has been performed in an arbitrary way and on the $X$-axis there are arbitrary units.

number of galaxies $\langle v(L, \boldsymbol{r})\rangle_{p} \mathrm{~d} \boldsymbol{r} \mathrm{d} L$ with luminosity in the range $[L, L+\mathrm{d} L]$ and in the volume element $\mathrm{d} \boldsymbol{r}$ at distance $r$ from an observer located on a galaxy. We can make the greatly simplifying assumption that

$\langle v(L, \boldsymbol{r})\rangle_{p}=\phi(L) \times\langle n(\boldsymbol{r})\rangle_{p}$,

where $\langle n(\boldsymbol{r})\rangle_{p}$ is the average conditional density and $\phi(L)$ the luminosity function, such that $\phi(L) \mathrm{d} L$ gives the probability that a randomly chosen galaxy has luminosity in the range $[L, L+\mathrm{d} L]$. Although there is clear evidence that there is a correlation between them and, for instance, that the brightest elliptical galaxies are found in the center of rich galaxy clusters, it has been tested that this is nevertheless a reasonable assumption in the galaxy catalogs available so far. To properly treat the case where the correlation between galaxy positions and luminosities is taken into account, one would need to use the multi-fractal formalism. This goes beyond the scope of the present manuscript (see Gabrielli et al. 2005). An additional, much stronger assumption often adopted in Eq. (13) is that the space density is a constant; i.e., $\langle n(\boldsymbol{r})\rangle=$ const. This assumption is for instance the basis of the so-called standard minimum variance estimator 


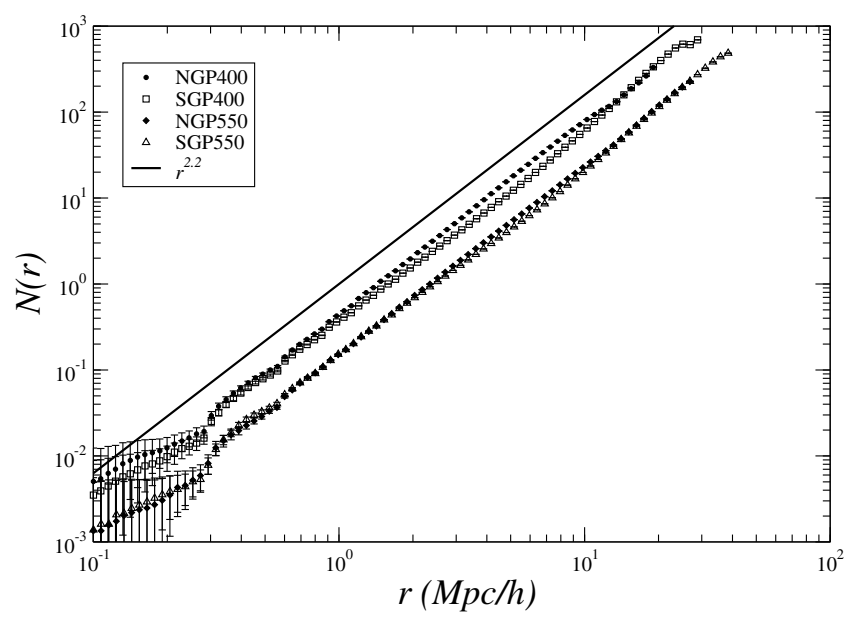

Fig. 12. Average number of points in spheres of radius $r$ around a galaxy. The difference amplitude in samples with different limits in absolute magnitude is simply ascribed by the effect of the luminosity function (see text). Error bars are estimated by the sample dispersion on the average value.

(Davis \& Huchra 1982). It is clear that we avoid making this further assumption because we want to test whether the space density is (or can be approximated by) a simple constant.

Using Eq. (13) we may write the conditional average number of galaxies as a function of distance (in case $D=$ const.)

$$
\begin{aligned}
& \left\langle N\left(r ; L_{V L}^{1}<L<L_{V L}^{2}\right)\right\rangle_{p} \\
& =\int_{0}^{r} \int_{L_{V L}^{1}}^{L_{V L}^{2}}\langle v(\boldsymbol{y}, L)\rangle_{p} \mathrm{~d} L \mathrm{~d} \boldsymbol{y} \\
& =B r^{D} \int_{L_{V L}^{1}}^{L_{V L}^{2}}\langle\phi(L)\rangle_{p} \mathrm{~d} L=B_{V L} r^{D}
\end{aligned}
$$

where $N\left(r ; L_{V L}^{1}<L<L_{V L}^{2}\right)$ is the number of galaxies in a sphere of radius $r$ and with intrinsic luminosity in the range $\left[L_{V L}^{1}, L_{V L}^{2}\right]$, and $B_{V L}$ is the amplitude of the number counts in the VL sample with these limits in absolute luminosity. Because the luminosity function has an exponential cut-off at $L^{*}$, VL samples containing brighter galaxies show a smaller $B_{V L}$. By knowing the shape of the luminosity function, it is simple to normalize the different $B_{V L}$ in different VL samples (Joyce \& Sylos Labini 2001).

\subsection{Average in radial bins}

We can now use the data obtained by the SL method to investigate whether there is a convergence to homogeneity on some large scales $r>30 \div 40 \mathrm{Mpc} / \mathrm{h}$; i.e., the largest sphere radius allowed by the geometry of the samples. This is done as follows. We divide the whole range of radial distances in bins of thickness $\Delta R$ and compute the average

$\overline{N(r ; R, \Delta R)}=\frac{1}{M_{b}} \sum_{R_{j} \in[R, \Delta R]}^{j=1, M_{b}} N\left(r ; R_{j}\right)$,

where the sum is extended to the $M_{b}$ determinations of $N(r ; R)$, such that the radial distance is in the interval range $[R, R+\Delta R]$. It is clear that for this measurement we have to use a small $r$ to

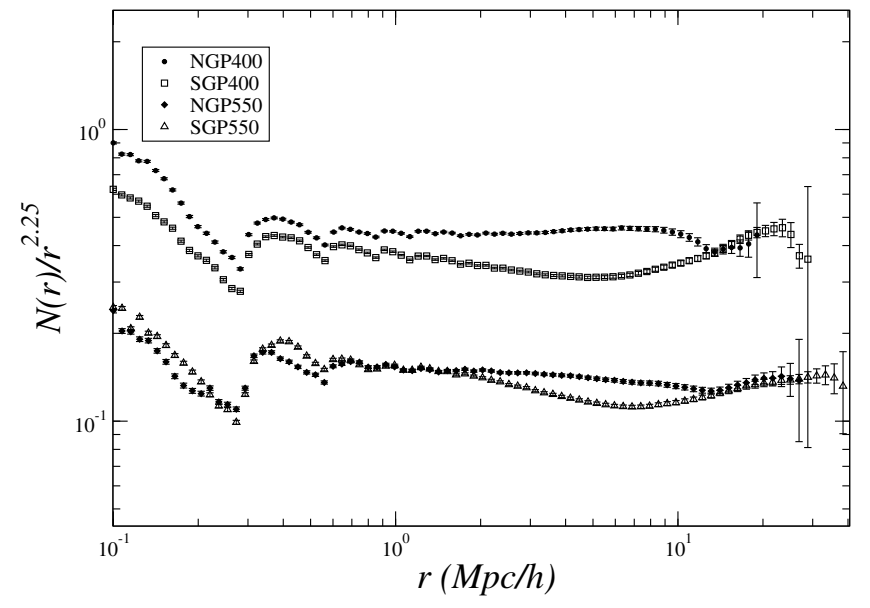

Fig. 13. The same as in Fig. 12 but divided by the best-fit power-law behavior $r^{2.25}$. The variation in the amplitude $B$ in Eq. (12) is clearer in this representation. The determination for $r>20 \mathrm{Mpc} / \mathrm{h}$ is subject to systematic fluctuations, due to the limited volume and the weaker statistics.

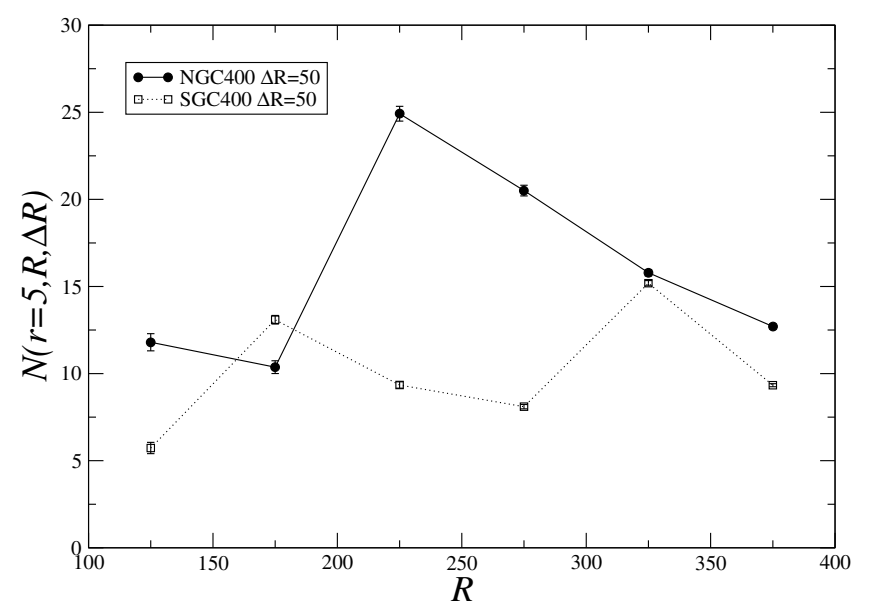

Fig. 14. Behavior of Eq. (15) with $r=5 \mathrm{Mpc} / \mathrm{h}$ for NGC400 and $\mathrm{SGC} 400$ and $\Delta R=50 \mathrm{Mpc} / \mathrm{h}$. The error is computed through Eq. (16).

avoid overlapping in space between neighboring bins in radial distance. The variance on the mean is

$\overline{\Sigma(r ; R, \Delta R)^{2}}=\sum_{R_{j} \in[R, \Delta R]}^{j=1, M_{b}} \frac{\left(N\left(r ; R_{j}\right)-\overline{N(r ; R, \Delta R)}\right)^{2}}{M_{b}\left(M_{b}-1\right)}$.

The error analysis in Eq. (16) assumes that the $N\left(r ; R_{j}\right)$ are independent, but in fact they are correlated. The errors on these points are therefore substantially under-estimated. However if there is a trend toward homogenization, the error caused by neglecting this correlation will be smaller hence the under estimate of the error bars. Only for the case of a highly correlated distribution does Eq. (16) underestimate the error bars, which than represent a lower limit of the "true" error bars.

The quantity given by Eq. (15) and its error (Eq. (16)) provide an estimation of the number of points in spheres of radius $r$ averaged in thickness bin $\Delta R$. We expect that, if the distribution converges to uniformity on a scale $\lambda_{0}$, then correspondingly $\overline{N\left(r ; R, \Delta R>\lambda_{0}\right)}$ does not show large fluctuations as a function of $R$.

Results for the four samples are shown in Figs. 14-15. One may note that, for the largest radial bin chosen $\Delta R=75 \mathrm{Mpc} / \mathrm{h}$, 


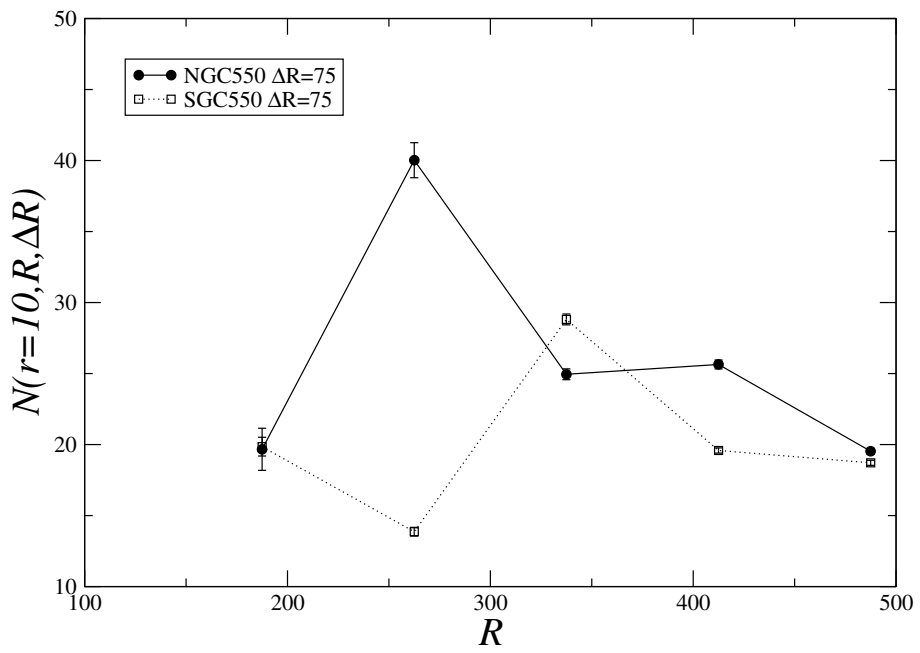

Fig. 15. As in Fig. 14 but for NGC550 and SGC550 with $r=10 \mathrm{Mpc} / \mathrm{h}$ and $\Delta R=50 \mathrm{Mpc} / \mathrm{h}$

there is no trend in homogenization, but instead the measurements in bins centered on different $R$ wildly scatters; i.e., their values are outside the statistical error bars given by Eq. (16). This shows that large-scale structures have an amplitude that is incompatible with homogeneity on scales smaller than $\lambda_{0}=\Delta R=$ $75 \mathrm{Mpc} / \mathrm{h}$.

It is interesting to note that the large fluctuations between the NGC and SGC samples cannot be generated by some redshiftdependent effect, such as the inclusion of galaxy evolution in the computation of absolute magnitudes. Indeed, such a correction, which is expected to be small anyway, given that the redshifts involved do not exceed 0.2 , would affect both samples in the same way. Thus by comparing the estimation of the density in bins in the same range of radial distances, we can conclude that the fluctuations we have detected are intrinsic to the distribution of galaxies in these samples. A similar argument can be made for the effect of different cosmologies in the computation of the metric distance.

\subsection{Radial counts in VL samples}

A complementary way to study fluctuations on large scales in galaxy redshift surveys is represented by the determination of the radial counts; i.e., the counts of galaxies as a function of the radial distance in VL samples (Gabrielli \& Sylos Labini 2001). In order to have a statistical estimator and to evaluate fluctuations, we divided the angular area of the samples into $N_{\mathrm{f}}=20$ non overlapping sub fields of equal solid angle. For each we compute the differential radial density $n_{i}(R ; \Delta R)$ in bins of thickness $\Delta R=10 \mathrm{Mpc} / \mathrm{h}$, where $i$ th labels the sub field and $R$ is, as usual, the radial distance. We then can compute the average

$\overline{n(R ; \Delta R)}=\frac{1}{N_{\mathrm{f}}} \sum_{i=1}^{N_{\mathrm{f}}} n_{i}(R ; \Delta R)$

and the sample variance

$\overline{\sigma_{n}^{2}(R ; \Delta R)}=\frac{1}{N_{\mathrm{f}}-1} \sum_{i=1}^{N_{\mathrm{f}}}\left(n_{i}(R ; \Delta R)-\overline{n(R ; \Delta R)}\right)^{2}$.

Results are shown in Figs. 16-17.

In the NGC400 sample the structure at $250 \mathrm{Mpc} / \mathrm{h}$ is visible as a relatively large fluctuation of $\overline{n(R ; \Delta R)}$ and a correspondingly large error. This means that this structure partially covers
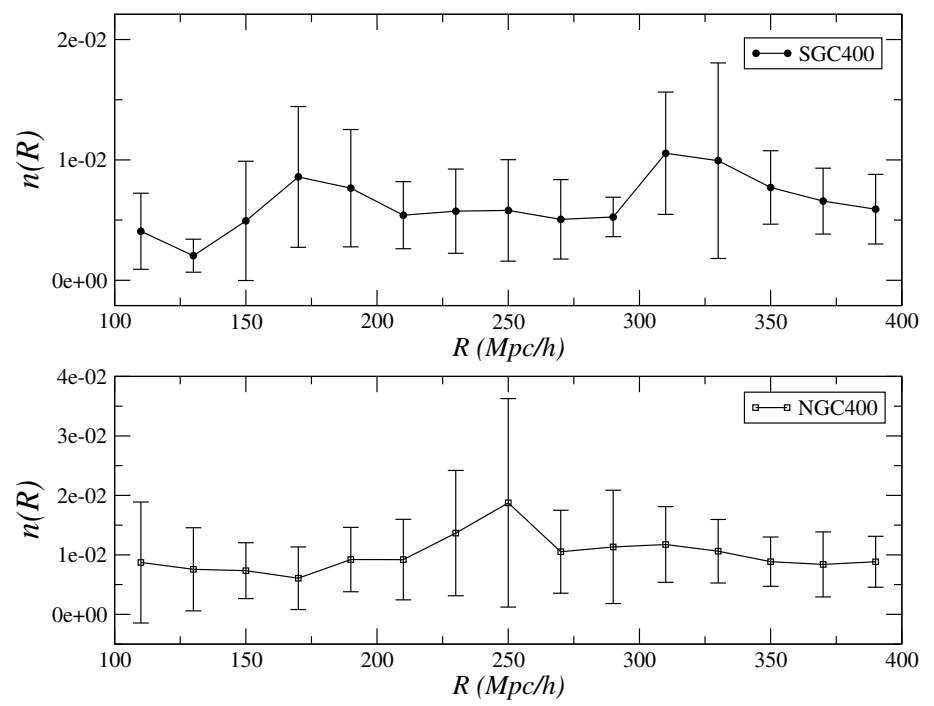

Fig. 16. The average differential radial density $\overline{n(R ; \Delta R)}$ (Eq. (17)) and its error (Eq. (18)) for the samples NGC400 and SGC400.
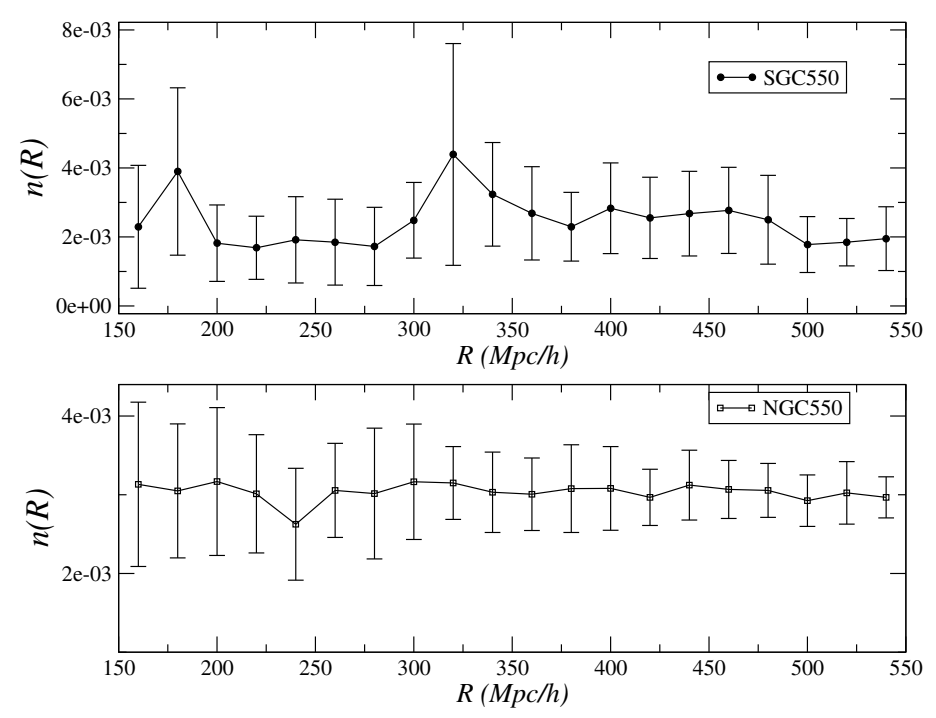

Fig. 17. The same as in Fig. 16 for SGC550 and NGC550.

the angular area of the survey. In the NGC550 sample, the radial density is flatter, although there is a large dispersion. In the SGC400 sample, the structures at $160 \mathrm{Mpc} / \mathrm{h}$ and $320 \mathrm{Mpc} / \mathrm{h}$ are identified as local enhancements of $\overline{n(R ; \Delta R)}$. The same occurs for the SGC550 case, where the same two structures are visible. By comparing Figs. 16-17 with Figs. 1-4 one may note that the SL analysis is a much more powerful method than the simple counting as a function of radial distance in tracing large-scale galaxy structures.

\subsection{Redshift distribution in the magnitude limit sample}

By studying the redshift distribution in the Durham/UKST Galaxy Redshift Survey, fluctuations have been found in the observed radial density function are close to $50 \%$ occurring on $\sim 50 \mathrm{Mpc} / \mathrm{h}$ scales (Ratcliffe et al. 1998; Busswell et al. 2004). In a similar way in the 2dFGRS (Busswell et al. 2004), two clear "holes" in the galaxy distribution were detected in the ranges $0.03<z<0.055$, with an under-density of $\sim 40 \%$, and $0.06<z<0.1$ where the density deficiency is $\sim 25 \%$. These two 

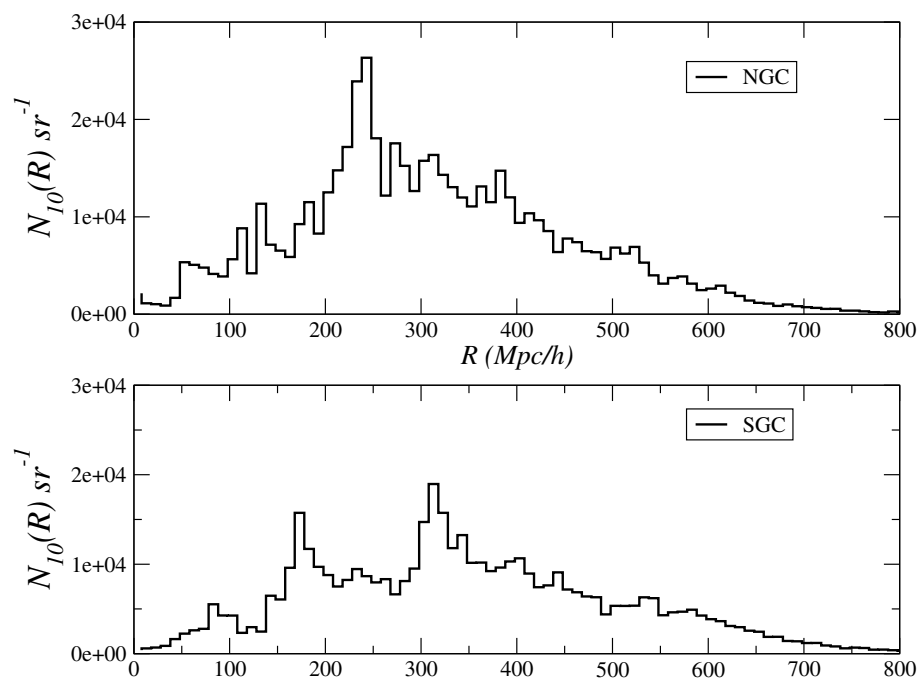

Fig. 18. Upper panel: radial density in bins of thickness $10 \mathrm{Mpc} / \mathrm{h}$ in the $N G C$ magnitude limited sample. The most prominent features identified by the $N(r ; R)$ analysis are also visible by the simple counting. There is a large structure at $\sim 240 \mathrm{Mpc} / \mathrm{h}$. By comparing this figure with Figs. 1-4 one may appreciate the usefulness of the SL method in tracing structures Bottom panel: the same for the SGC magnitude limited sample. Here there are large structures at $\sim 180 \mathrm{Mpc} / \mathrm{h}$ and $\sim 320 \mathrm{Mpc} / \mathrm{h}$.

under-densities, detected in particular in the 2dFGRS southern galactic cap (SGC), are also clear features in the Durham/UKST survey. Given that the 2dFGRS SGC field is entirely contained within the areas of sky observed for the Durham/UKST survey, the similarities in the redshift distributions are both proofs of the same features in the galaxy distribution (Busswell et al. 2004).

We can now compare the redshift distribution in the magnitude limit sample with the results obtained by the SL analysis. In Fig. 18 we report the counting of galaxies as a function of the radial distance, in bins of thickness $10 \mathrm{Mpc} / \mathrm{h}$, in the whole magnitude limited samples. It is interesting to compare these behaviors with Figs. 1-4. The SL method clearly identifies the same structures, which are visible in Fig. 18 as peaks of the radial distribution. However the SL method is able to quantify the amplitude of these fluctuations and, by applying the statistical analysis presented above, to determine how typical these structures are.

\subsection{Magnitude counts}

In Fig. 19 we report the differential counts of galaxies as a function of apparent magnitude in the SGC and NGC. The behaviors are similar to those found by Norberg et al. (2002a); Busswell et al. (2004): the former paper concluded that there is conclusive evidence that counts in the SGC are down by $30 \%$ relative to the NGC counts. We find the same difference and, as discussed, we can directly relate it to the large-scale structures present in both samples. Indeed, as already discussed, the amplitude of the conditional number of galaxies (i.e., Eq. (12)) is $\sim 20 \div 30 \%$ higher for the NGC samples than for the SGC ones. In other words, in the NGC samples there are more structures, hence fluctuations in the $N(r ; R)$, than in SGC samples, as can be seen by comparing, for instance, Figs. 1-3.

\subsection{The two-point correlation function}

The standard way to measure two-point correlations is accomplished by determining the function $\xi(r)$ given by Eq. (11) - see

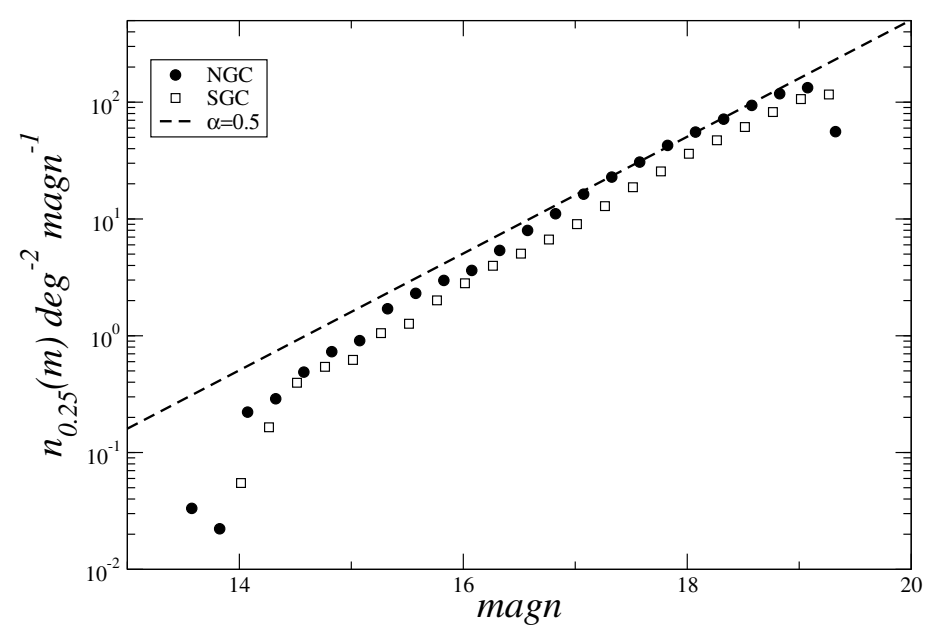

Fig. 19. Differential counts of galaxies, in bins of $\Delta m=0.25$, as a function of apparent magnitude in the SGC and NGC. A reference line corresponding to $N(m) \sim 10^{\alpha m}$ with $\alpha=0.5$ is reported.

e.g., Totsuji \& Kihara (1969), Davis \& Peebles (1983), Park et al. (1994), Benoist et al. (1996), Zehavi et al. (2002), Zehavi et al. (2004), Norberg et al. (2001), Norberg et al. (2002b). As already mentioned when measuring this quantity it is implicitly assumed that the distribution is homogeneous well inside the sample volume; i.e., $\lambda_{0} \ll V^{1 / 3}$. Let us see what happens when, inside a spherical sample of radius $R_{\mathrm{S}}$ there is a fractal distribution with dimension $D<3$. We may estimate the sample density $n_{\mathrm{S}}$ (which is not an average quantity) by

$n_{\mathrm{S}}=\frac{N}{V}=\frac{3 B}{4 \pi} R_{\mathrm{s}}^{D-3}$,

where, in the second equality on the rhs, we used Eq. (12) for the number of points in spheres. Equation (19) shows that the sample density depends on the sample size when $D<3$. The estimator of the two-point correlation function can be written as (Gabrielli et al. 2005)

$\overline{\xi(r)}+1=\frac{\overline{N(r, \Delta r)}}{V(r, \Delta r)} \cdot \frac{1}{n_{\mathrm{S}}}$

The first ratio in the rhs of Eq. (20) is the average conditional density; i.e., the number of galaxies in shells of thickness $\Delta r$ averaged over the whole sample, divided by the volume $V(r, \Delta r)$ of the shell. The second ratio in the rhs of Eq. (20) is the sample density. By using the second equality on the rhs of Eq. (19) for $n_{\mathrm{S}}$ and Eq. (12) for the conditional number of points in spheres, we find

$\overline{\xi(r)}=\frac{D}{3}\left(\frac{r}{R_{\mathrm{s}}}\right)^{D-3}-1$,

which shows that the amplitude of $\xi(r)$ depends on $R_{\mathrm{S}}$ for $D<$ 3 ; i.e., as long as the conditional density is a power law as a function of scale ${ }^{10}$.

Equation (21) has been obtained by making the assumption that the estimation of the sample density is given by the second equality in the rhs of Eq. (19). This is generallyot the case, as the sample density in inhomogeneous distributions is subjected

\footnotetext{
10 Pair-counting based estimators, like the Davis \& Peebles and/or the Landy \& Szalay estimator, have the same pathologies when the conditional density is a power law as a function of scale (Gabrielli et al. 2005).
} 
to fluctuations of order one on the scale of the sample size. Thus the behavior given by Eq. (21) should be interpreted as giving a very rough estimation of the amplitude of $\overline{\xi(r)}$ in a finite sample when there is a fractal distribution inside it.

It is worth noticing that, if the conditional density is a powerlaw function of scale, then $\overline{\xi(r)}$ is not a power-law over the same of scales of the conditional density, and particularly it does not have the same power law index. Indeed, as shown by Eq. (21), $\overline{\xi(r)}$ has a a break of the power law and it is possible to compute analytically the exponent of $\overline{\xi(r)}$ as a function of the exponent of the conditional density and the scale ratio $r / R_{\mathrm{S}}$ (Gabrielli et al. $2005)$. It is easy to show that on scales $r / R s \ll 1$ the correlation exponent measured by the two-point correlation analysis coincides with what is measured by the conditional density, while on larger scales the exponent measured by the $\xi(r)$ analysis is generally smaller than $D-3$. This is indeed the result obtained by Hawkins et al. (2003, see their Fig. 6).

Let us now evaluate the sample density simply as $n_{\mathrm{S}}=N / \mathrm{V}$ in SGC400 and NGC400. Given that the sample geometry is a sphere portion, the volume is given by

$V_{2 \mathrm{dFGRS}}=\frac{\Omega}{3}\left(R_{\max }^{3}-R_{\min }^{3}\right)$.

By using the parameters of the VL samples (see Table 1) we obtain respectively in the SGC400 sample

$\overline{n_{\mathrm{sgc}}}=7.0 \times 10^{-3}$

galaxies per $(\mathrm{Mpc} / \mathrm{h})^{3}$ and in the NGC400 sample

$\overline{n_{\text {ngc }}}=1.0 \times 10^{-2}$.

Thus there is a $\sim 30 \%$ difference in the amplitude. When we normalize the conditional density in the NGC400 and SGC400, which as discussed have the same $\sim 30 \%$ difference in amplitude, to the respective values of the sample density we get the amplitude of $\overline{\xi(r)}$ is nearly the same (see Fig. 20). This is because both the nominator and denominator in the rhs of Eq. (20) vary in the same way, having a difference of about $30 \%$ in both samples. Thus by measuring the amplitude of fluctuations normalized to the sample density the information about the large variations in the two different slices is lost. This does not, however, imply that fluctuations are small beyond $r_{0} \approx 6 \mathrm{Mpc} / \mathrm{h}$ as one would conclude by only considering the two-point correlation function. Actually, as we discussed in the previous sections, fluctuations are large and persistent over the whole volume of these samples. It is indeed the normalization of fluctuations amplitude to a not well-defined quantity, the sample density, that in this case gives rise to the determination of the length scale $r_{0} \approx 6 \mathrm{Mpc} / \mathrm{h}$, which is however a spurious quantity. The volume of the SGC400 sample is larger than that of the NGC400 sample: this is reflected in the fact that the break of $\overline{\xi(r)}$ occurs at a longer distance than for the NGC400 sample. For the samples NGC550 and SGC550, there is a similar situation, but the variation of both the sample density and of the amplitude of the conditional density is of about $20 \%$.

Different estimators of the two-point correlation function, such as the Davis and Peebles (DP) (Davis \& Peebles 1983) estimator and the Landy and Szalay (LS) (Landy \& Szalay 1993) estimator (see discussion in Sylos Labini \& Vasilyev (2008) for more details about the different estimators), lead to an estimation of the amplitude of $\overline{\xi(r)}$, which agrees with the one just discussed above. For instance in Fig. 21 a comparison is shown of

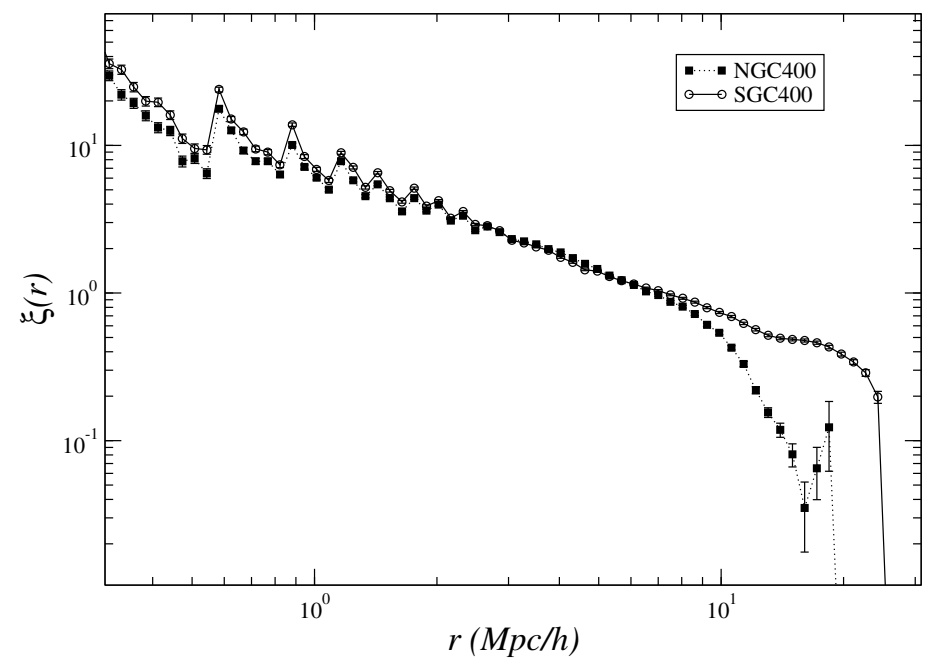

Fig. 20. Standard two-point correlation function in the SGC400 and NGC400 sample estimated by Eq. (20). The sample density is simply computed as $N / V$.

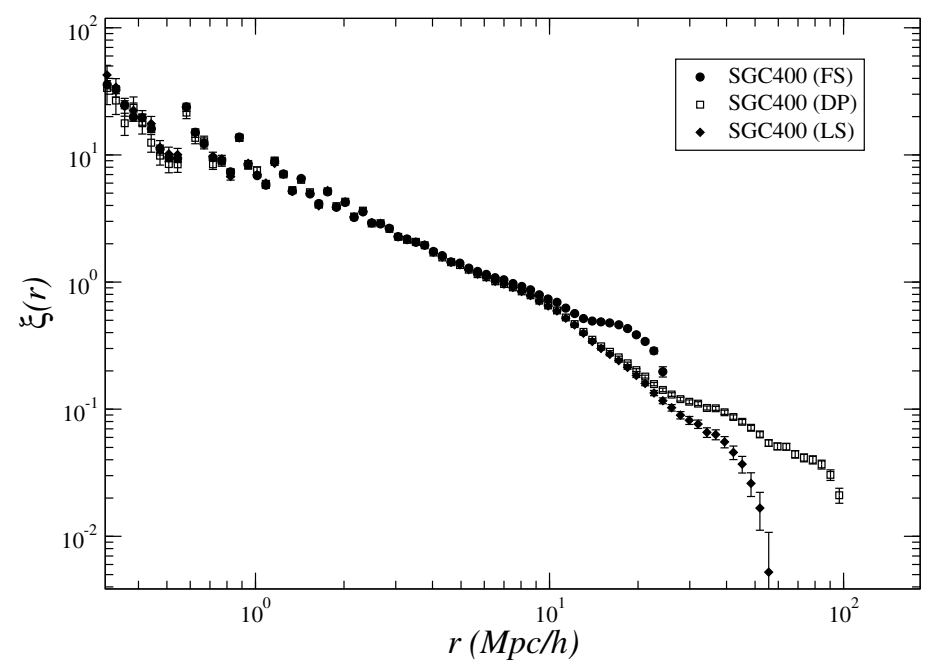

Fig. 21. Standard two-point correlation function in the SGC400 measured by means of different estimators, namely the full-shell (FS), the Davis and Peebles (DP) and the Landy and Szalay (LS).

the different estimators. It is worth noticing that the different estimators give the sample amplitude of $\overline{\xi(r)}$, but they differ in the scale at which $\overline{\xi(r)}$ has the break in the power-law behavior. This is explained by the different ways the estimators treat the boundary conditions, and, particularly, include (implicitly) the global condition known as integral constraint - see discussion in Sylos Labini \& Vasilyev (2008).

The sample density $n_{\mathrm{S}}$ can be estimated differently from Eq. (19). The only condition that it is required for Eq. (20) to be a valid estimator is that the size $r^{*}$ of the volume entering in the denominator of Eq. (19) be larger than the homogeneity scale $\lambda_{0}$. In fact, when $r^{*} \gg \lambda_{0}$ the estimation of the sample density does not differ substantially from its ensemble average value because, in this situation, the amplitude of the two-point correlation is, by definition, much smaller than unity. Thus we may consider another estimator of the two-point correlation function, which is the one introduced by Sylos Labini et al. (2008)

$\overline{\xi(r ; R, \Delta R)}+1=\frac{\overline{N(r, \Delta r)}}{V(r, \Delta r)} \cdot \frac{V(R, \Delta R)}{\overline{N(R, \Delta R)}}$, 


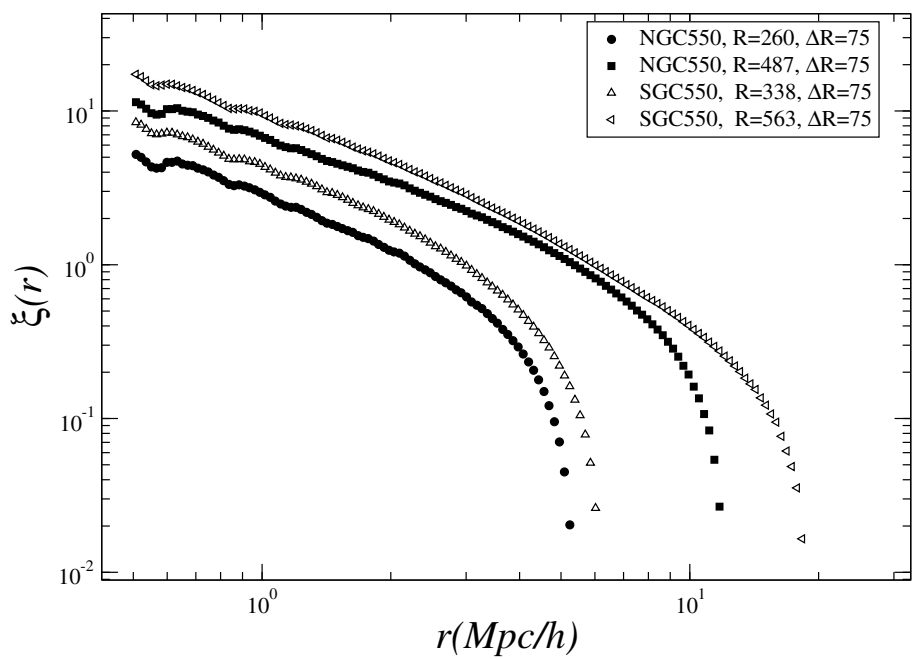

Fig. 22. Standard two-point correlation function in the SGC550 and NGC550 samples estimated by Eq. (23). The sample average density is computed in spheres of radius $r^{*}$ and considering all center points lying in a bin of thickness $\Delta R$ centered at different radial distances $R$.

where the second ratio on the rhs is the density of points in a shell of thickness $r^{*}=\Delta R$ around the radial distance $R$. If the distribution is homogeneous; i.e., $r^{*}>\lambda_{0}$, and statistically stationary, Eq. (23) should be statistically independent of the range of radial distances $(R, \Delta R)$ considered. In Fig. 22 we show the determination of Eq. (23) for different values of $R, \Delta R$ in the two deepest samples. The amplitude of $\overline{\xi(r)}$ systematically depends on the choice of the normalization and this is just the imprint of the large scale inhomogeneities present in these samples.

In summary, even though the fact that there is a relatively large difference in the densities between the NGC and the SGC, the amplitude of the correlation function is similar because it is measured with respect to a varying density; i.e., its value reflects the assumption of homogeneity which is used in the definition in the $\xi$-analysis. Only by analyzing fluctuations that are not normalized to the sample density one can detect the effect of the large spatial inhomogeneities characterizing these galaxy samples.

\subsection{Analysis in the catalog with conservative cuts in apparent magnitude}

We now briefly discuss the results of the SL analysis for the VL samples obtained with conservative magnitude cuts (see Table 2). In Fig. 23 we show the results of the SL analysis: by comparing this figure with Figs. 1-4 by a simple visual inspection one note that structures are extremely similar. Clearly, because of the fewer points contained in the conservative-cuts samples, the value of $N(r ; R)$ is different. The behavior of the PDF for the various samples is shown in Fig. 23. The results are thus statistically stable.

\section{Comparison with mock-catalogs}

Standard theories of galaxy formation assume that fluctuations in the matter density field in the early universe have very small amplitude, (Peebles 1980; Peacock 1999). In this situation, by denoting as $P(k, t)$, the PS of matter at an arbitrary time $t \gg 0$, where $t=t^{*}$ corresponds to some early times in the universe as

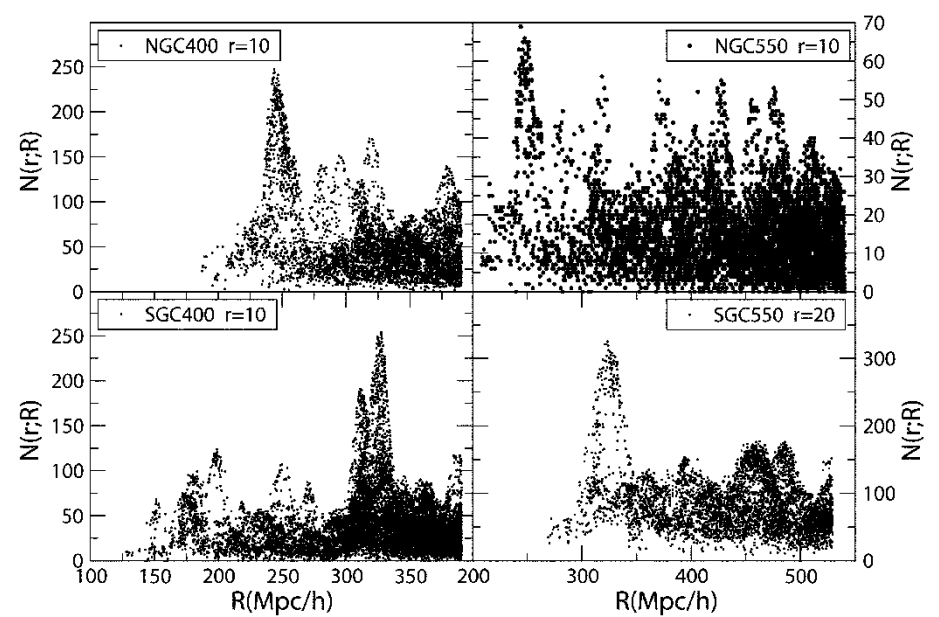

Fig. 23. SL analysis for the samples with conservative apparent magnitude cuts. The value of the sphere radius is reported in the captions.

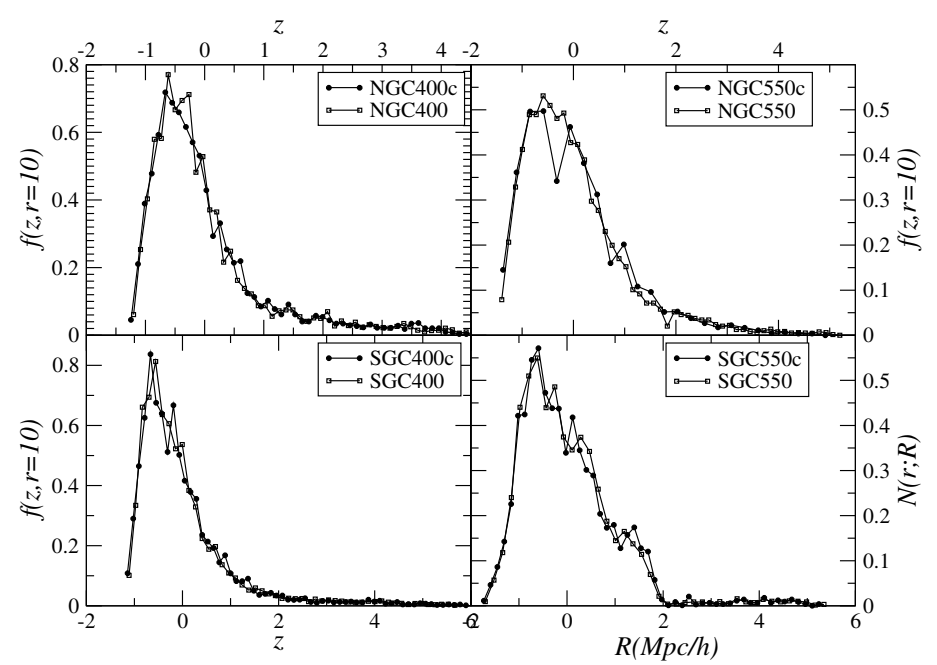

Fig. 24. PDF of the samples with conservative apparent magnitude cuts. The value of the sphere radius is reported in the labels. In the comparison we used the normalized variable $z$, by using the transformation described by Eqs. (26)-(27).

we discuss below, the prediction of standard cosmological models can be written in this form

$P(k, t)=A P\left(k, t^{*}\right) g(k, t)$.

It is possible to write $P\left(k, t^{*}\right)=P_{\mathrm{hz}}(k) T^{2}(k)$ where $P_{\mathrm{hz}}(k)$ is the Harrison-Zeldovich (HZ) PS, and $T(k)$ is a transfer function that depends on the type of coupling between matter and radiation in the early universe (Peacock 1999). The HZ PS is predicted by inflationary theories to be the outcome of the exponential amplification of quantum fluctuations occurring in the early phases after the Big-Bang, and this is such that $P_{\mathrm{hz}}(k) \propto k$. A PS behaving as $P(k) \propto k^{n}$ on small $k$ with $n \geq 1$ belonging to the class of super-homogeneous, or hyper-uniform (Torquato \& Stillinger 2003), distributions (Gabrielli et al. 2002). Their main characteristic is that density fluctuations are of surface type; i.e., that the relative mass variance shows the fastest possible decay as a function of scale $\sigma_{h z}^{2}(r) \propto 1 / r^{4}$. The PS must have a cut-off at some high values of $k$; i.e, on small scales. This is provided by the transfer function $T(k)$, which can be determined given the supposed properties of (dark) matter. For CDM this is such that at small $k$ the PS maintains the $\mathrm{HZ}$ tail, while at large $k$ it approximately decays as $k^{-2}$ (Peacock 1999). Correspondingly, 
the two-point correlation function is positive for $r<r_{\mathrm{c}}$ and negative for $r>r_{\mathrm{c}}$ with a power-law tail of the type $\xi(r) \sim-r^{-4}$ (Sylos Labini \& Vasilyev 2008).

Gravitational clustering in the linear regime, in an Einstein de Sitter cosmology, is characterized by a growing and a decaying mode, both of them power laws in time (Peebles 1980) $g(k, t)=g(t) \propto t^{4 / 3}$. The amplitude $A$ in Eq. (24) is determined from the observations of CMBR anisotropies and from the theoretical assumptions on the nature of cosmological dark matter. From the time dependence of $P(k, t)$, it is possible to derive the time dependence of $\lambda_{0}^{m}(t)$, defined to be $\sigma^{2}\left(\lambda_{0}^{m}\right)=1$. This grows as a power-law function of time as well: particularly for powerlaw PS; i.e., $P(k) \sim k^{n}$ and $n<4$, one obtains (Peebles 1980)

$\lambda_{0}^{m}(t) \propto t^{\frac{3}{3(3+n)}}$.

To summarize the situation, we may identify two length-scales at the present time. The first is the homogeneity (or nonlinearity) scale $\lambda_{0}^{m}$ : for $r<\lambda_{0}^{m}$ nonlinear clustering took place and thus it changes the shape of PS in a non-linear way. On the other hand for $r>\lambda_{0}^{m}$, fluctuations are still in the regime today, and linear perturbation theory predicts a simple linear amplification of primordial correlations. From the normalization of the initial amplitude $A$ to the CMBR anisotropies, $\lambda_{0}^{m} \approx 10 \mathrm{Mpc} / \mathrm{h}$ (Springel et al. 2005; Spergel et al. 2007). In addition, from the properties of the PS of CMBR anisotropies it is derived that the second intrinsic length scale of these models is about $r_{\mathrm{c}} \approx 100 \mathrm{Mpc} / \mathrm{h}$ (Sylos Labini \& Vasilyev 2008).

In order to study gravitational structure formation in the nonlinear phase, the common practice is to perform $N$-body simulations of theoretical models. This is done by integrating the equation of motions of $N$ self-gravitating particles, in a volume $V$ and by making use of periodic boundary conditions to represent an infinite (periodic) system. Initial particle correlations are given according to a given theoretical model, and the initial redshift is generally $z>10$. The simulation is then run up to $z=0$. In addition the space background is expanding and thus one follows the particles' motion in comoving coordinates. Particles are supposed to simulate the motion of fluid elements of the underlying dark matter field.

To identify galaxies one uses a phenomenological approach. As discussed, galaxies are supposed to form in the highest density peaks of the dark matter field. Thus when the simulation has reached the redshift $z=0$, one uses semi-analytic models to identify galaxies. Among the largest simulations made publicy available, the millennium run (Springel et al. 2005) used more than 10 billion particles to trace the evolution of the matter distribution in a region of the universe in a cubic box of $500 \mathrm{Mpc} / \mathrm{h}$. Semi-analytic catalogs constructed from the millennium run contains about 10 million objects (Croton et al. 2006).

Here we analyze the semi-analytic catalog containing 9925229 objects in which the absolute magnitudes of mockgalaxies are given in the $B V R I K$ filters. To reproduce the same limits in absolute magnitude of the volume-limited samples of the $2 \mathrm{dFGRS}$, we used the relations between magnitude in different filters given by Colless et al. (2001). In this way we selected respectively 1119434 and 368619 galaxies in a $500 \mathrm{Mpc} / \mathrm{h}$ cube. We then selected three slices with the same geometry of the real 2dFGRS samples, which are hereafter called SGC400m, NGC400m and NGC550m. The remaining sample, SGC550m, is $60^{\circ}$ wide instead of $84 \circ$ as in real data. The number of objects in each of the four samples is close to the one in the corresponding real 2dFGRS sample.

In Fig. 25 we show the behavior of the SL analysis for the mock-sample SGC550m in real and redshift space for

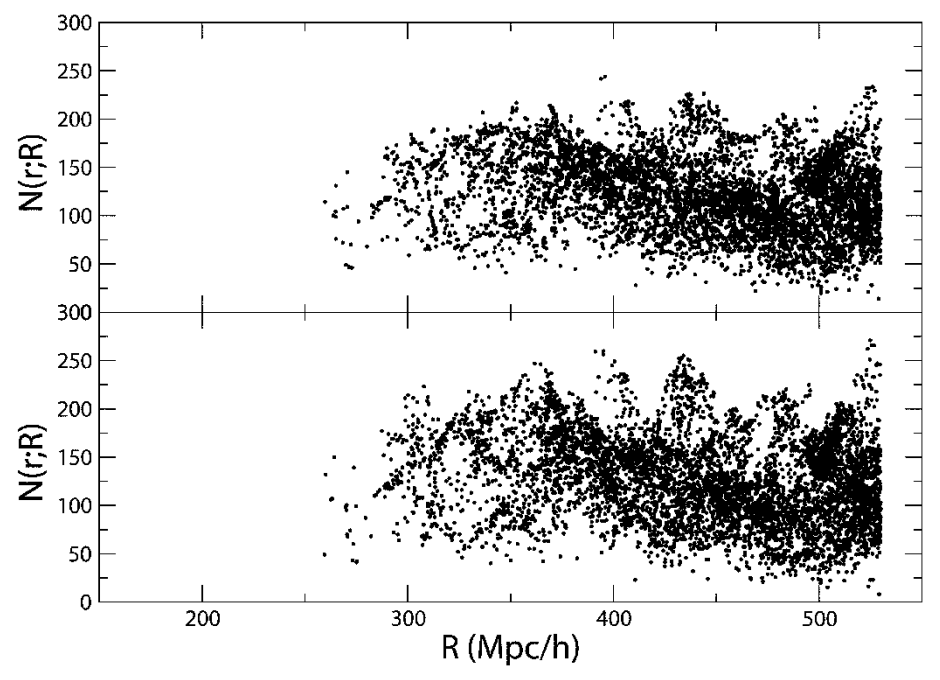

Fig. 25. SL analysis with sphere radius $r=20 \mathrm{Mpc} / \mathrm{h}$, for the mocksample SGC550m (the samples with better statistics) in real space (upper panel) and in redshift space (bottom panel).

$r=20 \mathrm{Mpc} / \mathrm{h}$ (see for comparison Figs. 2-4). The effect of peculiar velocities is that of enhancing a little the structures that appear in real space. That the difference between real and redshift space is small for sphere radius $r \geq 10 \mathrm{Mpc} / \mathrm{h}$ is shown in Figs. 26-27, where we plot the PDF of conditional fluctuations. In this case we used the normalized variable

$z_{i}(r)=\frac{N_{i}(r)-\overline{N(r)}}{\overline{\Sigma(r)}}$

and we thus determine its PDF, that is,

$P\left(z_{i}, r\right)=f\left(N_{i}(r)=\overline{N(r)}+z_{i} \overline{\Sigma(r)}\right) \times \overline{\Sigma(r)}$

where $f(N, r)$ is the PDF of the variable $N_{i}(r), \overline{N(r)}$ its estimated first moment, and $\overline{\Sigma(r)}$ its estimated standard deviation on the scale $r$. For sphere radius smaller than the homogeneity scale predicted by theoretical models; i.e., $\lambda_{0}^{m}=10 \mathrm{Mpc} / \mathrm{h}$, the PDF has a relatively large tail for high values of $N$, although around the peak it is well fitted by Gaussian function. For $r>10 \mathrm{Mpc} / \mathrm{h}$, the PDF rapidly converges to a Gaussian and already for $r=$ $20 \mathrm{Mpc} / \mathrm{h}$ the fit with a Gaussian function is extremely good also at high $N$. Correspondingly the plot of the SL analysis does not show the presence of large amplitude fluctuations.

In Figs. 28-29 we show the comparison between the PDF in the mock-sample SGC500m (redshift space) and the one in the real sample SGC550. Both for the sphere radius $r=10 \mathrm{Mpc} / \mathrm{h}$ and $r=20 \mathrm{Mpc} / \mathrm{h}$, fluctuations are more persistent in real samples than in mock-samples and this is reflected in the relatively fat tail of the PDF for large $N$ values.

In Fig. 30 (upper panel) it is shown the behavior of the whole-sample average number of points is spheres; i.e., Eq. (5). For $r>\lambda_{0}^{m}=10 \mathrm{Mpc} / \mathrm{h}$, the function $\overline{N(r)}$ growths with an exponent equal to the space dimension (i.e., $D=3$ ). On smaller scales the exponent is instead $D=1.2 \pm 0.1$.

Finally in the Fig. 30 (bottom panel) the behavior of of Eq. (15) with $r=5 \mathrm{Mpc} / \mathrm{h}$ is shown for the mock-galaxy samples in real space. Fluctuations, when averaged over radial bin of thickness $\Delta R=50 \mathrm{Mpc} / \mathrm{h}$ are more smooth and quiet than the real samples and thus that there are no large fluctuations on scales larger than $\lambda_{0}^{m}$. This is clearly consistent with the behavior of the PDF described previously. The small difference for 


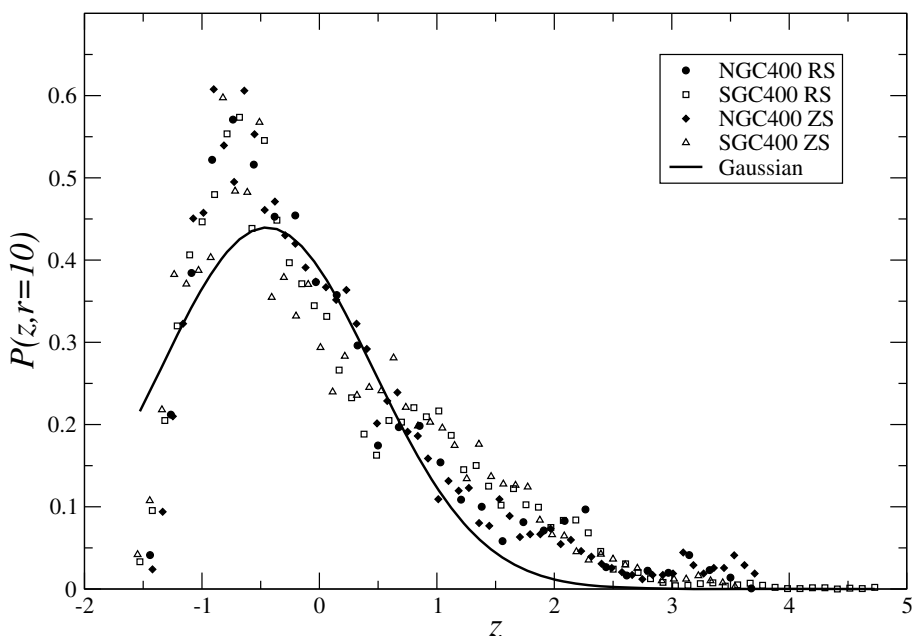

Fig. 26. PDF for the mock-samples SGC400m and NGC400m in real space (RS) and redshift space (ZS) for $r=10 \mathrm{Mpc} / \mathrm{h}$. The best fit with a Gaussian function is reported as reference.

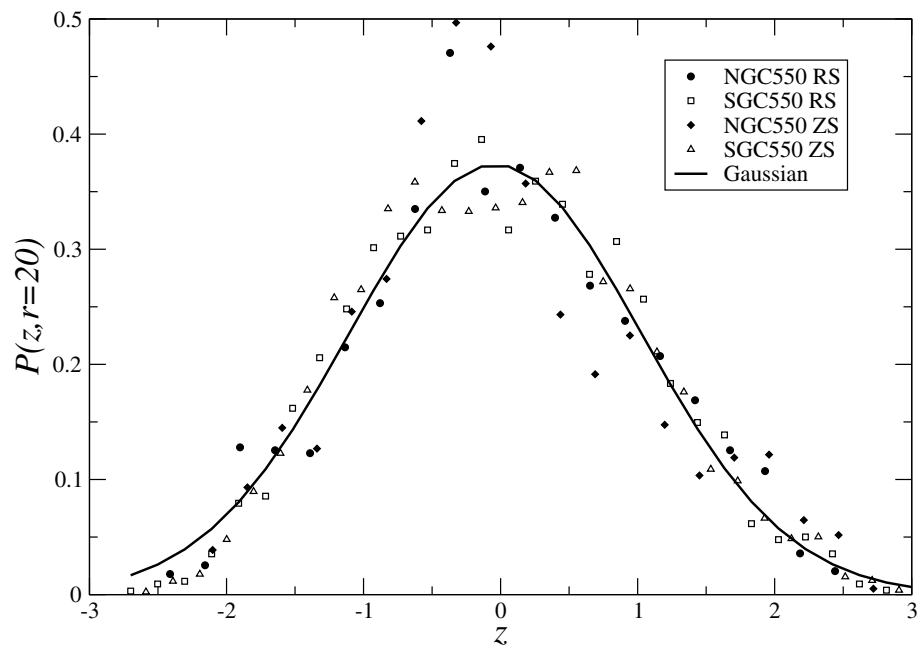

Fig. 27. The same as in Fig. 26 but for the case $r=20 \mathrm{Mpc} / \mathrm{h}$ and for the mock-samples SGC550m and NGC500m.

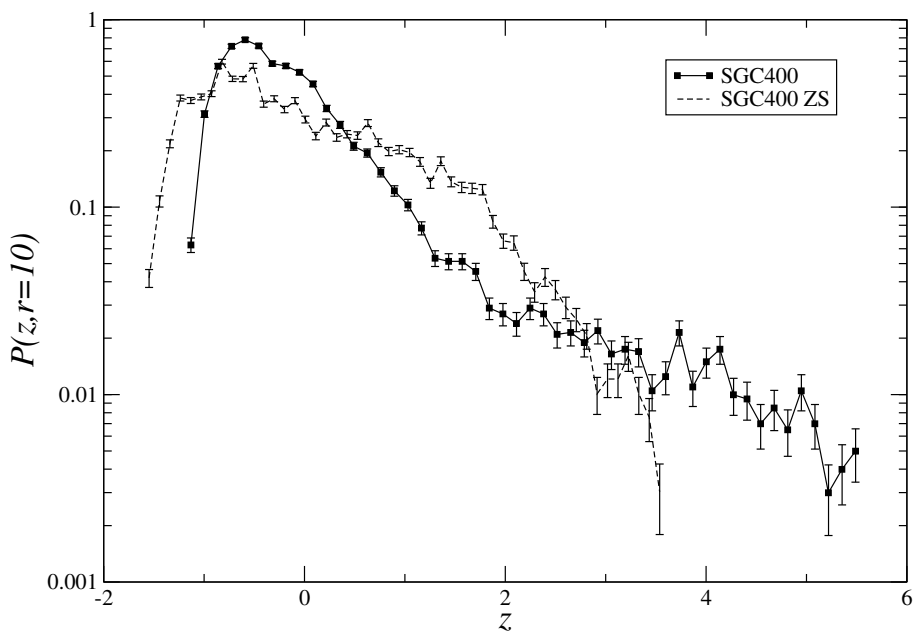

Fig. 28. Comparison between the PDF in the mock-sample SGC400m (redshift space) and the one in the real sample SGC400. Poisson error bars are displayed as a reference.

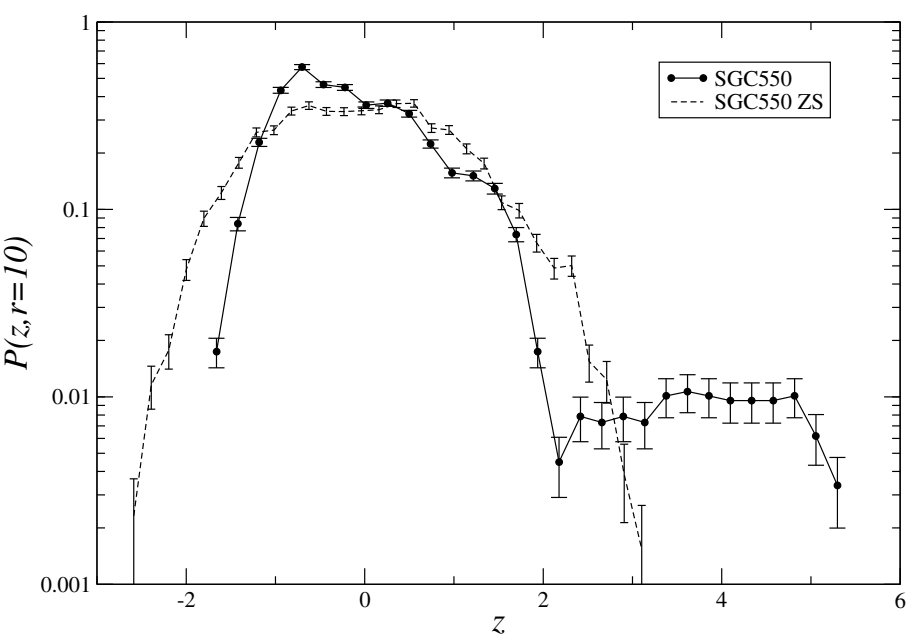

Fig. 29. The same as in Fig. 28 but for the case $r=20 \mathrm{Mpc} / \mathrm{h}$ and for the sample SGC550.
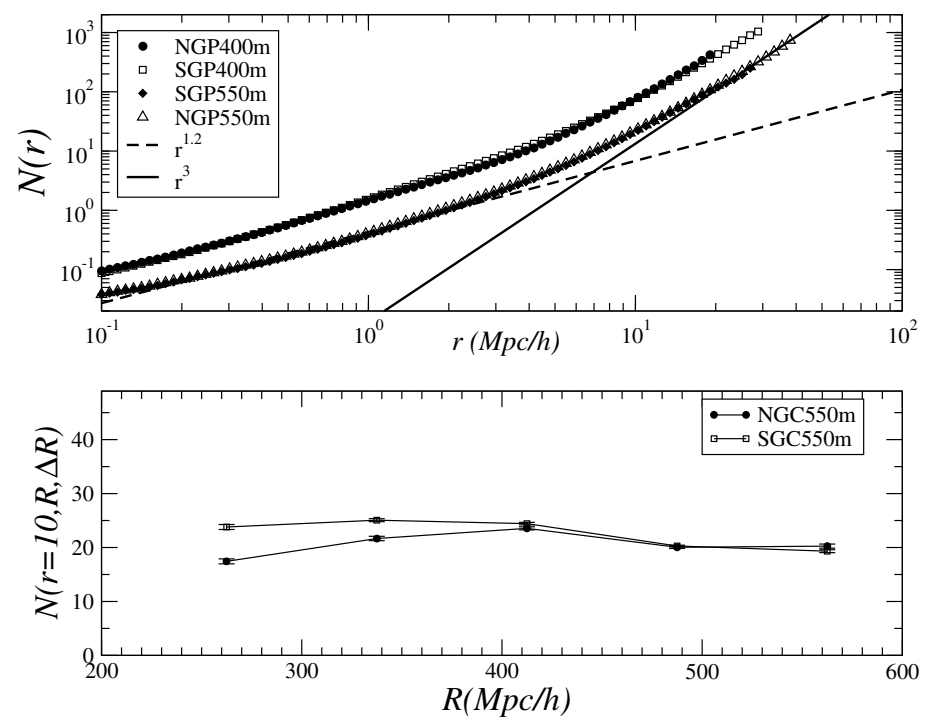

Fig. 30. Upper panel: whole-sample average number of points in spheres; i.e., Eq. (5) for the mock-samples in real space. Bottom panel: behavior of Eq. (15) for the mock-galaxy samples in real space for SGC550m; the sphere radius is $r=10 \mathrm{Mpc} / \mathrm{h}$ and the radial bin thickness is $\Delta R=75 \mathrm{Mpc} / \mathrm{h}$. The error is computed through Eq. (16).

small $R$ is due to the lower statistics at the nearby boundary of the samples.

Because the distribution becomes uniform at scales $r>\lambda_{0} \approx$ $10 \mathrm{Mpc} / \mathrm{h}$, the two-point correlation function is well-defined, both in real and redshift space. This presents an intrinsic length scale $\xi\left(r_{0}\right)=1$ at about $r_{0} \approx 6 \mathrm{Mpc} / \mathrm{h}$, and beyond that scale it rapidly decays to zero.

The whole-sample average number of points in spheres (i.e., Eq. (5)) does show a systematic difference with respect to the real-space case. Indeed, while for $r>\lambda_{0}^{m}=10 \mathrm{Mpc} / \mathrm{h}$ it again shows a power-law behavior with $D=3$, on smaller scales the exponent is $D=2.2 \pm 0.1$ instead of $D=1.2$ as for the real space case (see Fig. 31 - upper panel). In addition, the transition between the two regimes is faster than for the real space case.

Finally in Fig. 31 (bottom panel) the behavior of Eq. (15) is reported for the mock-samples in redshift space. As for the real-space case, and consistent with the behaviors of the PDF 

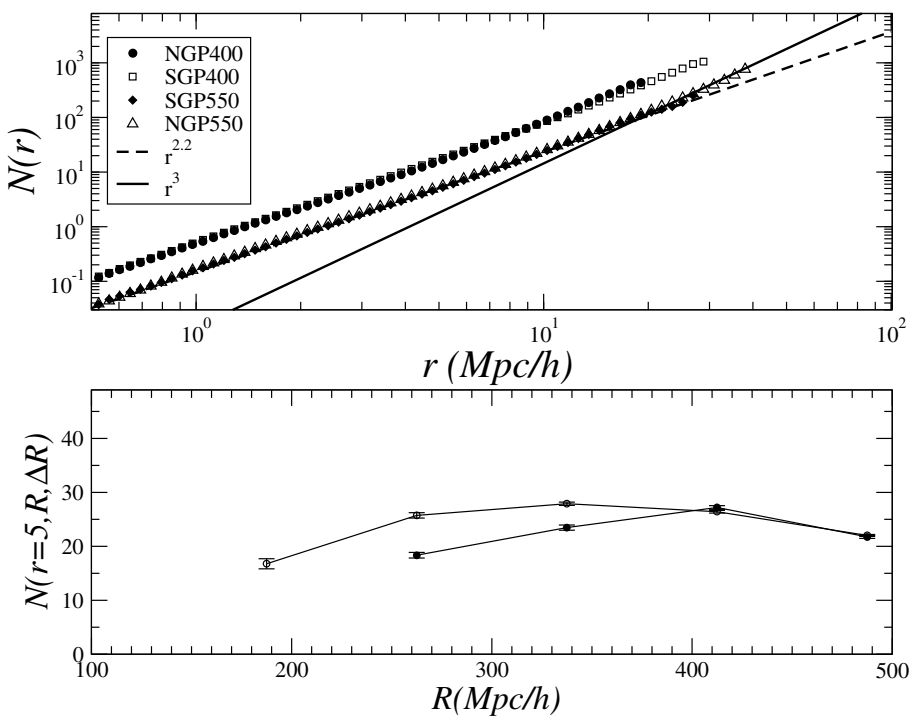

Fig. 31. Upper panel: whole-sample average number of points in spheres; i.e., Eq. (5). for the mock-samples in redshift space. Bottom panel: behavior of Eq. (15) for the mock-samples in redshift space for SGC550m; the sphere radius is $r=10 \mathrm{Mpc} / \mathrm{h}$ and the radial bin thickness is $\Delta R=75 \mathrm{Mpc} / \mathrm{h}$. The error is computed through Eq. (16).

in redshift space, fluctuations are substantially smoothed when averaged over radial bins of size $\Delta R>\lambda_{0}^{m}$.

\section{Discussion and conclusions}

We characterized fluctuations and correlations in the galaxy distribution, by considering several samples from the $2 \mathrm{dFGRS}$ (Colless et al. 2001). The main point of our analysis is to use statistical quantities that measure fluctuations amplitude not normalizing it to the sample average. Our main results are:

- Estimations of the sample average are affected by systematic variations that are the imprint of the structures with the large spatial extension present in these samples, which correspond to large amplitude fluctuations. To identify structures in redshift space we applied the scale-length (SL) method, introduced in Sylos Labini et al. (2008).

- The probability density function (PDF) of conditional fluctuations is, in all considered samples and up to $r=20 \mathrm{Mpc} / \mathrm{h}$ (the largest distance scale we considered for this statistics), wildly different from a Gaussian function and characterized by a long tail, possibly with a power-law decay, signaling the presence of large fluctuations (e.g. structures) in the density field. These fluctuations are persistent up to scales limited by sample sizes.

- The first moment of the PDF shows scaling properties, that correspond to a fractal dimension $D=2.2 \pm 0.1$ up to $r \approx 40 \mathrm{Mpc} / \mathrm{h}$. At the largest distance scale we considered; i.e., for $r>20 \mathrm{Mpc} / \mathrm{h}$, determinations are affected by relatively large fluctuations that reflect the intrinsic systematic error induced by structures, and thus the results have weaker statistical significance for the limited volume and number of points contributing to the average. This agrees with our previous findings in Vasilyev et al. (2006).

- The homogeneity scale, i.e. the scale beyond which fluctuations become small and the sample density estimation approaches the value of the (ensemble) average density, has a lower limit at $\lambda_{0}=70 \mathrm{Mpc} / \mathrm{h}$. This is because fluctuations on scales of about $70 \mathrm{Mpc} / \mathrm{h}$ are incompatible with a transition to homogeneity on that scale. This result is consistent with the results by Busswell et al. (2004); Ratcliffe et al. (1998). The main results obtained in this survey are in very good agreement with a similar analysis of galaxy samples extracted from the SDSS (Sylos Labini et al. 2008).

- The SL method is suitable and effective in tracing structures in redshift space and in measuring the amplitude and distribution of density fluctuations. Galaxy counts results can be easily related to the results obtained by the SL method. Indeed we confirmed that the variation of about $\sim 30 \%$ between galaxy counts as a function of apparent magnitude in the northern and southern angular regions of this survey, detected by Busswell et al. (2004), can be easily related to the different structures present in the different samples. Moreover, such large fluctuations, make any estimation of the sample average, on comparable scales to the samples sizes, affected by strong systematic effects.

- Apparently the amplitude the two-point correlation function, which normalizes the amplitude of fluctuations (as a function of scale) to the estimation of the sample average, of is not affected by fluctuations, and that this is stable in the Northern and Southern samples. This occurs, however, because of the normalization to the sample average (i.e., the denominator of $\xi(r))$ which indeed shows the same large variation between the two angular regions as the conditional density (i.e., the nominator of $\xi(r)$ ). Thus the normalization of fluctuations amplitude to the sample average hides the presence of large differences between the two samples, being biased by uncontrolled systematic finite-size effects. In this situation the amplitude of the two-point correlation function does not give a reliable and meaningful estimation of either the statistical properties of galaxy distribution in these samples, the spatial extension of correlations, or of amplitude of fluctuations. It is indeed the normalization of fluctuations amplitude to a not well defined quantity, the sample density, which in this case determines the spurious length scale $r_{0} \approx 6 \mathrm{Mpc} / \mathrm{h}$.

- We compared the results by studying conditional fluctuations in the 2dFGRS samples with those obtained in mock-galaxy catalogs. These are constructed from the dark matter density field in cosmological simulations which were evolved to study the effect of non-linear gravitational clustering in an expanding universe. We used the mock-galaxy samples provided by Croton et al. (2006) and obtained from the largest $N$-body simulations publicly available at the moment (Springel et al. 2005). We have studied the mock-galaxy catalogs both in real and redshift space, so to take into account the distortions due to the effect of peculiar velocities. We found that the PDF of conditional fluctuations is weakly affected by peculiar velocities and that it rapidly converges to a Gaussian function on scales $r>10 \mathrm{Mpc} / \mathrm{h}$, in agreement with predictions of theoretical models both in real and redshift space. On smaller scales, i.e. $r<10 \mathrm{Mpc} / \mathrm{h}$, the PDF presents a longer tail than the Gaussian function. This is the signature of the nonlinear structures formed at small scales. Indeed, the PDF of mock-galaxy catalogs reflect the dynamics that has given rise to structures in the simulations. In this respect, correlations provided by theoretical models and given as initial conditions for the simulations are of CDM type. In these models gravitational clutering forms nonlinear structures (i.e., large fluctuations in the density field) up to the limited range of about $10 \mathrm{Mpc} / \mathrm{h}$. Such a length scale can be easily predicted by considering the initial amplitude of fluctuations in these models, normalized to CMBR 
anisotropies, and by computing the evolution in an expanding universe of the linear regime of gravitational clustering. Note that Einasto et al. (2006) reached a similar conclusion; i.e., that in the $2 \mathrm{dFGRS}$ there are several rich super-clusters which is higher than what is found in similar volumes in the Millennium simulation.

Our conclusion is therefore that nonlinear structures (i.e., large fluctuations) predicted by models extend to scales that are much smaller than the ones we found in the 2dFGRS sample and that amplitude of fluctuations on large scales, i.e., $r>10 \mathrm{Mpc} / \mathrm{h}$, is large in the real samples and small in the mock-galaxy catalogs. Thus standard models of galaxy formation, with CMBR normalization, are unable to form large-scale structures such as the ones present in the observed galaxy distribution. In addition, standard models, beyond the value of the homogeneity scale, predict a second intrinsic length scale represented by the distance scale at which the two-point correlation function changes sign (Durrer et al. 2003; Sylos Labini \& Vasilyev 2008); i.e., marking the transition from small amplitude fluctuations with weak positive correlations to the regime where there are anti-correlations (and fluctuations of extremely small amplitude). This length scale is about $r_{\mathrm{c}} \approx 100 \mathrm{Mpc} / \mathrm{h}$ in current models; i.e., slightly larger than the lower limit to the homogeneity scale $\lambda_{0}=70 \mathrm{Mpc} / \mathrm{h}$ we can place by studying the $2 \mathrm{dFGRS}$ samples. Forthcoming galaxy samples will allow us to determine whether there will be a transition to super-homogeneity (negative correlations) and on which scale this eventually occurs.

Acknowledgements. We thank Michael Joyce, Andrea Gabrielli, and Luciano Pietronero for useful remarks and discussions. We are grateful to Martin LopezCorreidoira and Michael Blanton for interesting comments. We also thank an anonymous referee for useful suggestions. Yu.B. thanks the RF grant "Leading Scientific School" for partial financial support. We acknowledge the use of the 2dFGRS data available at http://www.mso.anu.edu.au/2dFGRS/ and of the millennium run semi-analytic galaxy catalog available at http://www . mpa-garching.mpg.de/galform/agnpaper/

\section{References}

Baryshev, Yu., \& Teerikorpi, P. 2006, Bull. Spec. Astrop. Obs., 59, 92 Benoist, C., Maurogordato, S., da Costa, L. N., Cappi, A., \& Schaeffer, R. 1996, ApJ, 472, 452

Busswell, G. S., Shanks, T., Frith, W. J., et al., 2004, MNRAS, 354, 991

Chiaki, H., Schmalzing, J., Buchert, T., et al. 2003, PASJ, 55, 911

Colless, M., Dalton, G., Maddox, S., et al. 2001, MNRAS, 328, 1039

Croton, D. J., Springel, V., White, S. D. M., et al. 2006, MNRAS, 365, 11

da Costa, L., Pellegrini, P. S., Sargent, W. L. W., et al. 1988, ApJ, 327, 544

Davis, M., \& Huchra, J. 1982, ApJ, 254, 437
Davis, M., \& Peebles, P. J. E. 1983, ApJ, 267, 465

Davis, M., Meiksin, A., Strauss, M. A., et al. 1988, ApJ, 333, L9 de Lapparent, V., Geller, M. J., \& Huchra, J. P. 1986, ApJ, 302, 1 de Vaucouleurs, G. 1970, Science, 167, 1203

Durrer, R., Gabrielli, A., Joyce, M., \& Sylos Labini, F. 2003, ApJ, 585, L1

Eke, V. R., Baugh, C. M., Cole, S., et al. 2004, MNRAS, 348, 866

Einasto, J., Einasto, M., Saar, E., et al. 2006, A\&A, 459, L1

Falco, E. F., Kurtz, M. J., Geller, M. J., et al. 1999, PASP, 111, 438

Frith, W. J., Busswell, G. S., Fong, R., Metcalfe, N., \& Shanks, T. 2003, MNRAS, 345, 1049

Gabrielli, A., \& Sylos Labini, F. 2001, Europhys. Lett., 54, 286

Gabrielli, A., Joyce, M., \& Sylos Labini, F. 2002, Phys. Rev. D, 65, 083523

Gabrielli, A., Sylos Labini, F., Joyce, M., \& Pietronero, L. 2005 Statistical

Physics for Cosmic Structures (Berlin: Springer Verlag)

Geller, M., \& Huchra J. 1989, Science, 246, 897

Giovanelli, R., \& Haynes, M. P. 1993, AJ, 105, 1271

Gott, J., Richard, III, Juric', M., \& Schlegel, D. 2005, ApJ, 624, 463

Hawkins, Ed, Maddox, S., Cole, S., et al. 2003, MNARS, 346, 78

Hogg, D. W., Eisenstein, D., Blanton, M., et al. 2005, ApJ, 624, 54

Hogg, D. W., Baldry, I. K., Blanton, M. R., \& Eisenstein, D. J. 2002, [arXiv: astro-ph/0210394v1]

Huchra, D., Latham, \& Tonry, 1983, ApJS 52, 89

Kaiser, N. 1984, ApJ, 284, L9

Kerscher, M., Schmalzing, J., Buchert, T., \& Wagner, H. 1998, A\&A, 333, 1

Kerscher, M. 1999, A\&A, 343, 333

Joyce, M., \& Sylos, L. F. 2001, ApJ, 554, L1

Landy, S. D., \& Szalay, A. 1993, ApJ, 412, 64

Madgwick, D. S., Lahav, O., Baldry, I. K., et al. 2002, MNRAS, 333, 133

Norberg, P., Baugh, C. M., Hawkins, Ed., et al. 2001, MNRAS, 328, 64

Norberg, P., Cole, S., Baugh, C. M., et al. 2002a, MNRAS, 336, 907

Norberg, P., Baugh, C. M., Hawkins, Ed., et al. 2002b, MNRAS, 332, 827

Park, C., Vogeley, M. S., Geller, M. J., \& Huchra, J. P. 1994, ApJ, 431, 569

Peacock, J. A. 1999, Cosmological physics (Cambridge University Press)

Peebles, P. J. E. 1980, The Large-Scale Structure of the Universe (Princeton University Press)

Picard, A. 1991, AJ, 102, 445

Ratcliffe, A., Shanks, T., Parker, Q. A., \& Fong, R., 1998, MNRAS, 293, 197

Saslaw, W. C. 2000, The Distribution of the Galaxies, (Cambridge: Cambridge University Press)

Shectman, S. A., Landy, S. D., Oemler, A. et al. 1996, ApJ, 470, 172

Spergel, D. N., Bean, R., Doré, O., et al. 2007, ApJS, 170, 377

Springel, V., White, S. D. M., Jenkins, A., et al. 2005, Nature, 435, 629

Sylos Labini, F., Montuori, M., \& Pietronero, L. 1988, Phys. Rep., 293, 61

Sylos Labini, F., Vasilyev, N. L., \& Baryshev, Yu. V. 2007, A\&A, 465, 23

Sylos Labini, F., Vasilyev, N. L., Pietronero, L., Baryshev, \& Yu. V. 2008, [arXiv:0805.1132v1]

Sylos Labini, F., \& Vasilyev, N. L. 2008, A\&A, 477, 381

Sylos Labini, F., Vasilyev, N. L., \& Baryshev, Yu. V. 2009, Europhys. Lett., 85, 29002

Torquato, S., \& Stillinger, F. H. 2003, Phys. Rev. E, 68, 041113

Totsuji, H., \& Kihara, T. 1969, PASJ, 21, 221

Vasilyev, N. L., Baryshev, Yu. V., \& Sylos Labini, F., 2006, A\&A, 447, 431

York, Donald, G., Adelman, J., Anderson, J. E., Jr., et al. 2000, AJ, 120, 1579

Weir, N., Djorgovski, G. S., \& Fayyad, U. M. 1995, AJ, 110, 1

Wu, K. K., Lahav, O., \& Rees, M. 1999, Nature, 225, 230

Zehavi, I., Blanton, M. R., Frieman, J. A., et al. 2002, ApJ, 571, 172

Zehavi, I., Weinberg, D. H., Zheng, Z., et al. 2004, ApJ, 608, 16

Zehavi, I., Eisenstein, D. J., Nichol, R. C., et al. 2005, ApJ, 621, 22 